\title{
Sources of alpha-, beta-, gamma-, delta- and epsilon-carotenes: A twentieth century review
}

\author{
José M. Barbosa-Filho, * Adriana A. Alencar, Xirley P. Nunes, Anna C. de Andrade Tomaz, \\ José G. Sena-Filho, Petrônio F. Athayde-Filho, Marcelo S. Silva, Maria F. Vanderlei de \\ Souza, Emidio V. Leitão da-Cunha
}

\author{
Laboratório de Tecnologia Farmacêutica, Universidade Federal da Paraíba, Caixa Postal 5009, \\ 58051-970 João Pessoa-PB, Brazil
}

\begin{abstract}
RESUMO: "Fontes de alfa-, beta-, gama-, delta-, e epsilon-carotenos: Uma revisão do século XX". Uma vez que os humanos não podem sintetizar carotenóides, dependem exclusivamente da dieta como fonte desses micronutrientes. Tem sido afirmado que eles podem aliviar doenças crônicas, tais como cânceres. O presente artigo constitui uma revisão global da literatura científica sobre plantas e outros organismos que biossintetizam carotenóides, que incluem as séries alfa-, beta-, gama-, delta-, e epsilon-carotenos. Os resultados das listas de pesquisa da literatura mostram mais de quinhentas fontes.
\end{abstract}

Unitermos: $\alpha$-Caroteno, $\beta$-caroteno, $\gamma$-caroteno, $\delta$-caroteno, $\varepsilon$-caroteno, carotenóides, câncer, revisão.

\begin{abstract}
Since humans cannot synthesize carotenoids, they depend upon the diet exclusively for the source of these micronutrients. It has claimed that they may alleviate chronic diseases such as cancers. The present communication constitutes a global review of the scientific literature on plants and others organisms that biosynthesize carotenoids, which include the series alpha-, beta-, gamma-, delta- and epsilon-carotenes. The results of the literature survey lists more than five hundred sources.
\end{abstract}

Keywords: $\alpha$-Carotene, $\beta$-carotene, $\gamma$-carotene, $\delta$-carotene, $\varepsilon$-carotene, carotenoids, cancer, review.

\section{INTRODUCTION}

The carotenoids constitute the largest class of naturally occurring pigments and have attracted the attention of chemists and biologists since 1831, when an yellow coloring agent named $\beta$-carotene was isolated from the carrot (Daucus carota) for the first time. Many treatises have been devoted to the almost 1000 cis-trans carotenoid isomers characterized in nature (Britton, 1984, 1985, 1986, 1989, 1991). Most have 40 carbon atoms, less than $10 \%$ are hydrocarbons, most have oxygen functions, and some are conjugated with sugars and other molecules. Major provitamin A in mammals are $\beta$-carotene and $\alpha$-carotene, although $\gamma$-carotene and approximately 50 other carotenoids also show some nutritional activity. Carotenoids are synthesized in plants from acetyl-coenzyme A by a series of well-defined condensations reactions. The synthesis of carotenoids does not occur in animal tissue; however, when those pigments are ingested in the diet, the animals deposit or modify their basic structure (Olson, 1989).

Most cancers that are common nowadays are not caused by the products of modern industry, but by the lifestyle, especially the diet. The scientific literature is consistent in evidences that it is possible to reduce the risk of acquiring some types of cancer by eating a variety of vegetables and fruits (Steinmetz \& Potter, 1996; Federmann \& Federmann, 2000). The types of vegetable or fruit that most often appear to be protective against cancer are raw vegetables, followed by allium, carrots, green vegetables, cruciferous vegetables, and tomatoes. Substances present in vegetables and fruit that may help protect against cancer, and their mechanisms, include, allium compounds, flavonoids, protease inhibitors, saponins, phytosterols, carotenoids, vitamins, selenium and dietary fiber. Increment in vegetable and fruit consumption, include also benefits against cardiovascular disease, diabetes, stroke, obesity, diverticulosis, and cataracts (Peto et al., 1981).

In a previous paper this research group has reviewed crude plant extracts and chemically defined molecules with potential antitumor activity for mammary (Moura et al., 2001), cervical (Moura et al., 2002) and ovarian neoplasias (Silva et al., 2003), as inhibitors of HMG CoA reductase (Gonçalves et al., 2000), central analgesic activity (Almeida et al., 2001), employed in 
prevention of osteoporosis (Pereira et al., 2002), for the treatment of Parkinson's disease (Morais et al., 2003), with antileishmanial (Rocha et al., 2005), hypoglycemic (Barbosa-Filho et al., 2005), antiinflammatory activity (Falcão et al., 2005, Barbosa-Filho et al., 2006a), inhibitors of the enzyme acetylcholinesterase (BarbosaFilho et al., 2006b), inhibitors of the angiotensin converting enzymes (Barbosa-Filho et al., 2006c), giardicidal (Amaral et al., 2006), and antileprotic activity (Barbosa-Filho et al., 2007).

This work aims at presenting the main natural sources of carotenoids, which include the series $\alpha-, \beta$-, $\gamma$ -,$\delta$ - and $\varepsilon$-carotene.

\section{MATERIAL AND METHODS}

An extensive literature search was carried out using the Chemical Abstract, Biological Abstract and in the data bank of the University of Illinois in Chicago - NAPRALERT (Acronym for NAtural PRoducts ALERT), updated to March 2000.

The keywords used (in various combinations) in the search were: plants natural products, carotenoids, alpha-, beta-, gamma-, delta- and epsilon-carotene. The references found in the search were then consulted.

Consultation of various types of literature resulted in the elaboration of a list of occurrence of alpha-, beta-, gamma-, delta- and epsilon-carotene in plants and other organisms. For details the original references should be consulted.

\section{Source of carotenoids}

Carotenoids are found almost everywhere in nature, but particularly among organisms that bask in the sun. Those interesting compounds, most of which show a yellow to red color, have attracted the attention of chemists and biologists since at least the early $1800 \mathrm{~s}$. The colored compounds in plants and microorganisms were extracted and purified, and in time their structures were determined. Many treatises have been devoted to these compounds (Isler, 1971) the most comprehensive revision were presented by Briton (Britton, 1984, 1985, 1986, 1989, 1991).

We encountered 690 carotenes reported in the literature, 477 are found to be $\beta$-carotene, $144 \alpha$-carotene, $43 \gamma$-carotene, $22 \varepsilon$-carotene and $4 \delta$-carotene, so 310 are found in Dicotyledons, 122 Monocotyledons, 76 algae, 44 Gymnosperms, 22 ferns and allies, 8 lichens, 5 fungi, 4 bacteria and 2 insects.

Orange vegetables, such as carrots, sweet potatoes, winter squash, and pumpkin, are relatively rich sources of $\beta$-carotene, as are some fruits, including papaya, mango and cantaloupe. Because of the interest of carotenoids in preventing many types of cancer, it is also important to know which specific plants keep the greatest number of them. We found that Calendula arvensis, C. officinalis, Citrus paradise, Daucus carota and Diospiros kaki biosynthesize $\alpha-, \beta-, \gamma-\delta$ - and $\varepsilon$-carotenes (Table 1).<smiles>CC1=CCCC(C)(C)C1/C=C/C(C)=C/C=C/C(C)=C/C=C/C=C(C)/C=C/C=C(C)/C=C/C1=C(C)CCCC1(C)C</smiles><smiles>CC1=C(/C=C/C(C)=C/C=C/C(C)=C/C=C/C=C(C)/C=C/C=C(C)/C=C/C2=C(C)CCCC2(C)C)C(C)(C)CCC1</smiles><smiles>CC(C)=CCC/C(C)=C/C=C/C(C)=C/C=C/C(C)=C/C=C/C=C(C)/C=C/C=C(C)/C=C/C1=C(C)CCCC1(C)C</smiles><smiles>CC1=CCCC(C)(C)C1/C=C/C(C)=C/C=C/C(C)=C/C=C/C=C(C)/C=C/C=C(C)/C=C/C=C(\C)CCC(C)=C(C)C</smiles><smiles>CC1=CCCC(C)(C)C1/C=C/C(C)=C/C=C/C(C)=C/C=C/C=C(C)/C=C/C=C(C)/C=C/[C@H]1C(C)=CCCC1(C)C</smiles>

Figure 1. Chemical structure of carotene series. 
Table 1. Summary of distribution of natural carotene series.

\begin{tabular}{|c|c|c|c|c|c|c|c|c|}
\hline \multirow[b]{2}{*}{ Species } & \multirow[b]{2}{*}{ Family } & \multirow[b]{2}{*}{ Group } & \multicolumn{5}{|c|}{ Carotene series } & \multirow[b]{2}{*}{ Reference } \\
\hline & & & $\alpha-C$ & $\beta-C$ & $\gamma-\mathrm{C}$ & $\delta-\mathrm{C}$ & $\varepsilon-\mathrm{C}$ & \\
\hline Acanthopanax sp & Araliaceae & $\mathrm{D}$ & & $\sqrt{ }$ & & & & Zorikov \& Burii, 1974 \\
\hline Acanthophora spicifera & Rhodomelaceae & $\mathrm{D}$ & & $\sqrt{ }$ & & & & Aihara \& Yamamoto, 1968 \\
\hline Acidum aculeatum & Aspleniaceae & FE & $\sqrt{ }$ & $\sqrt{ }$ & & & & Czeczuga, 1985b \\
\hline Acidum angulare & Aspleniaceae & $\mathrm{FE}$ & $\sqrt{ }$ & $\sqrt{ }$ & & & & Czeczuga, $1985 b$ \\
\hline Adiantum capillus-veneris & Pteridaceae & $\mathrm{FE}$ & $\sqrt{ }$ & $\sqrt{ }$ & & & & Czeczuga, $1985 b$ \\
\hline Adiantum pedatum & Pteridaceae & FE & $\sqrt{ }$ & & & & & Czeczuga, $1985 \mathrm{~b}$ \\
\hline Adiantum venustum & Pteridaceae & $\mathrm{FE}$ & $\sqrt{ }$ & $\sqrt{ }$ & & & & Czeczuga, $1985 b$ \\
\hline Aesculus hippocastanum & Hippocastanaceae & $\mathrm{D}$ & $\sqrt{ }$ & & & & & Souleles \& Vayas, 1986 \\
\hline Agastache foeniculum & Lamiaceae & $\mathrm{D}$ & & $\sqrt{ }$ & & & & Menghini et al.,1992 \\
\hline Aglais urticae & Nymphalidae & I & & $\sqrt{ }$ & & & & Kayser, 1975 \\
\hline Alectoria nigricans & Parmeliaceae & $\mathrm{L}$ & $\sqrt{ }$ & $\sqrt{ }$ & & & & Czeczuga \& Olech, 1990 \\
\hline Allium cepa & Liliaceae & M & & $\sqrt{ }$ & & & & Granado, 1992 \\
\hline Aloe vera & Liliaceae & M & & $\sqrt{ }$ & & & & Rowe \& Parks, 1941 \\
\hline Amaranthus tricolor & Amaranthaceae & $\mathrm{D}$ & & $\sqrt{ }$ & & & & Rao, 1967 \\
\hline Amblystegium riparium & Amblystegiaceae & $\mathrm{FE}$ & $\sqrt{ }$ & $\sqrt{ }$ & & & & Czeczuga, 1980a \\
\hline Anabaena hallensis & Nostocaceae & $\mathrm{A}$ & & $\sqrt{ }$ & & & & Hai et al., 1996 \\
\hline Anacystis nidulans & Chroococcaceae & A & & $\sqrt{ }$ & & & & Buchecker et al., 1976 \\
\hline Ananas comosus & Bromeliaceae & M & $\sqrt{ }$ & $\sqrt{ }$ & & & & Morgan,1966 \\
\hline Aneura pinguis & Aneuraceae & $\mathrm{FE}$ & & $\sqrt{ }$ & & & & Benesova, 1969 \\
\hline Angelica tatianae & Apiaceae & $\mathrm{D}$ & & $\sqrt{ }$ & & & & Aprikyan, 1979 \\
\hline Anisomorpha buprestoides & Bacunculidae & I & & $\sqrt{ }$ & & & & Davidson, 1991 \\
\hline Ankistrodesmus braunii & Oocystaceae & A & & $\sqrt{ }$ & & & & Burczyk, 1987 \\
\hline Anthurus archeri & Clathraceae & FU & & & & & $\sqrt{ }$ & Czygan, 1975 \\
\hline Aphanizomenon flos-aquae & Nostocaceae & A & & $\sqrt{ }$ & & & & Hertzberg \& Jensen, 1966 \\
\hline \multirow[t]{2}{*}{ Apium graveolens } & Apiaceae & $\mathrm{D}$ & & $\sqrt{ }$ & & & & Fraczek et al., 1977 \\
\hline & & & & & & & & Gupta et al., 1980 \\
\hline Aplysia fasciata & Aplysiidae & S & & $\sqrt{ }$ & & & & Czeczuga, 1984 \\
\hline Aplysia rosea & Aplysiidae & $\mathrm{S}$ & $\sqrt{ }$ & $\sqrt{ }$ & & & & Czeczuga, 1984 \\
\hline Arabidopsis thaliana & Brassicaceae & $\mathrm{D}$ & & $\sqrt{ }$ & & & & Parry \& Horgan, 1992 \\
\hline Argania spinosa & Sapotaceae & $\mathrm{D}$ & & $\sqrt{ }$ & & & & Collier \&Lemaire, 1974 \\
\hline Arnica montana & Asteraceae & $\mathrm{D}$ & $\sqrt{ }$ & $\sqrt{ }$ & & & & Vanhaelen, 1973 \\
\hline Aronia melanocarpa & Rosaceae & $\mathrm{D}$ & & $\sqrt{ }$ & & & & Razungles et al., 1989 \\
\hline \multirow[t]{2}{*}{ Artemisia dracunculus } & Asteraceae & $\mathrm{D}$ & & $\sqrt{ }$ & & & & Prodan et al., 1973 \\
\hline & & & & & & & & Trofimova, 1977 \\
\hline Artemisia sylvatica & Asteraceae & $\mathrm{D}$ & & $\sqrt{ }$ & & & & Moon et al., 1999 \\
\hline Arum maculatum & Araceae & M & & $\sqrt{ }$ & & & & Valadon \& Mummery, 1975 \\
\hline Ascidia zara & Ascidiidae & $\mathrm{S}$ & & $\sqrt{ }$ & & & & Ookubo \& Matsuno, 1985 \\
\hline Ascophyllum nodosum & Fucaceae & $\mathrm{A}$ & & $\sqrt{ }$ & & & & Haugan \& Jensen, 1994 \\
\hline \multirow[t]{3}{*}{ Asparagus officinalis } & Liliaceae & M & $\sqrt{ }$ & $\sqrt{ }$ & & & & Simpson et al., 1977 \\
\hline & & & & & & & & Bureau \& Bushway, 1986 \\
\hline & & & & & & & & Granado et al., 1992 \\
\hline Aspidium lobatum & Dryopteridaceae & $\mathrm{FE}$ & $\sqrt{ }$ & $\sqrt{ }$ & & & & Czeczuga, 1985b \\
\hline Asplenium lanceolatum & Aspleniaceae & $\mathrm{FE}$ & $\sqrt{ }$ & $\sqrt{ }$ & & & & Czeczuga, 1985b \\
\hline Asplenium trichomanes & Aspleniaceae & $\mathrm{FE}$ & $\sqrt{ }$ & & & & & Czeczuga, 1985b \\
\hline Asterias amurensis & Asteriidae & $\mathrm{S}$ & & $\sqrt{ }$ & & & & Maoka et al., 1989 \\
\hline Asterina pectinifera & Asterinidae & $\mathrm{S}$ & & $\sqrt{ }$ & & & & Maoka et al., 1989 \\
\hline Athyrium filix-femina & Dryopteridaceae & $\mathrm{FE}$ & & $\sqrt{ }$ & & & & Czeczuga, 1985b \\
\hline Averrhoa carambola & Oxalidaceae & $\mathrm{D}$ & & $\sqrt{ }$ & & & $\sqrt{ }$ & Gross, 1983 \\
\hline Basella alba & Basellaceae & $\mathrm{D}$ & & $\sqrt{ }$ & & & & Cyunel \& Czygan, 1989 \\
\hline Berberis amurensis & Berberidaceae & $\mathrm{D}$ & & $\sqrt{ }$ & & & & Shapiro, 1983 \\
\hline Berberis integerrima & Berberidaceae & $\mathrm{D}$ & & $\sqrt{ }$ & & & & Shapiro, 1983 \\
\hline Berberis koreana & Berberidaceae & $\mathrm{D}$ & & $\sqrt{ }$ & & & & Shapiro, 1983 \\
\hline Berberis sieboldii & Berberidaceae & $\mathrm{D}$ & & $\sqrt{ }$ & & & & Shapiro, 1983 \\
\hline Berberis thunbergii & Berberidaceae & $\mathrm{D}$ & & $\sqrt{ }$ & & & & Shapiro, 1983 \\
\hline Berberis vulgaris & Berberidaceae & $\mathrm{D}$ & & $\sqrt{ }$ & & & & Shapiro, 1983 \\
\hline \multirow[t]{2}{*}{ Beta vulgaris } & Chenopodiaceae & $\mathrm{D}$ & $\sqrt{ }$ & $\sqrt{ }$ & & & & Granado et al., 1992 \\
\hline & & & & & & & & Bureau \& Bushway, 1986 \\
\hline \multirow[t]{2}{*}{ Bixa orellana } & Bixaceae & $\mathrm{D}$ & & $\sqrt{ }$ & & & & Angelucci et al., 1980 \\
\hline & & & & & & & & Tirimanna, 1981 \\
\hline Blakeslea trispora & Choanephoraceae & FU & & $\sqrt{ }$ & $\sqrt{ }$ & & & Feofilova \& Red'kina, 1975 \\
\hline & & & & & & & & Feofilova, 1974 \\
\hline Blechnun occidentale & Blechnaceae & $\mathrm{FU}$ & $\sqrt{ }$ & $\sqrt{ }$ & & & & Czeczuga, 1985b \\
\hline Boehmeria nivea & Urticaceae & $\mathrm{D}$ & $\sqrt{ }$ & $\sqrt{ }$ & & & & Santos, 1980 \\
\hline Botrylloides violaceus & Styelidae & $\mathrm{S}$ & & $\sqrt{ }$ & & & & Ookubo \& Matsuno, 1985 \\
\hline & & & & & & & & Matsuno \& Sakaguchi, 1984 \\
\hline Botryllus schlosseri & Styelidae & S & & $\sqrt{ }$ & & & & Ookubo \& Matsuno, 1985 \\
\hline Brassica chinensis & Brassicaceae & $\mathrm{D}$ & & $\sqrt{ }$ & & & & Ortaliza, 1969 \\
\hline
\end{tabular}




\begin{tabular}{|c|c|}
\hline Species & Family \\
\hline $\begin{array}{l}\text { Brassica napus } \\
\text { Brassica oleracea }\end{array}$ & $\begin{array}{l}\text { Brassicaceae } \\
\text { Brassicaceae }\end{array}$ \\
\hline Bromelia fastuosa & Bromeliaceae \\
\hline Bryocaulon divergens & Stereocaulaceae \\
\hline Bufo calamita & Bufonidae \\
\hline Caesalpinia japonica & Fabaceae \\
\hline Calendula arvensis & Asteraceae \\
\hline Calendula officinalis & Asteraceae \\
\hline
\end{tabular}

\begin{tabular}{c|c|c|c|c|c|}
\multirow{3}{*}{ Group } & \multicolumn{5}{|c|}{ Carotene series } \\
\cline { 2 - 6 } & $\alpha-\mathrm{C}$ & $\beta-\mathrm{C}$ & $\gamma-\mathrm{C}$ & $\delta-\mathrm{C}$ & $\varepsilon-\mathrm{C}$ \\
\hline $\mathrm{D}$ & \multicolumn{5}{c}{$\sqrt{ }$} \\
\hline
\end{tabular}

$\sqrt{ }$ Joyce, 1954

Granado et al., 1992

Bureau \& Bushway, 1986

Pospisilova, 1959

Takagi, 1985

Khachik et al., 1999

Wu et al., 1992

Bauer \& Demirovska, 1982

Bobbio \& Scamparini, 1980

Czeczuga \& Olech, 1990

Czeczuga, 1983

Imamura et al., 1980

Baraud, 1958

Calendula officinalis Asteraceae

$\begin{array}{llll} & \sqrt{ } & & \\ \sqrt{ } & \sqrt{ } & \sqrt{ } & \sqrt{ } \\ \sqrt{ } & \sqrt{ } & \sqrt{ } & \end{array}$

Camellia sasanqua

Cantharellus cibarius

Cantharellus lateritus

Cantharellus tabernensis

Capparis decidua

Capparis zeylanica

Capsicum annuum

Theaceae

Cantharellaceae

Cantharellaceae

Cantharellaceae

Capparidaceae

Capparidaceae

Solanaceae

$\begin{array}{cccc}\text { D } & \sqrt{ } & & \\ \text { FU } & \sqrt{ } & \sqrt{ } & \sqrt{ } \\ \text { FU } & \sqrt{ } & \sqrt{ } & \\ \text { FU } & \sqrt{ } & \sqrt{ } & \sqrt{ } \\ \text { D } & & \sqrt{ } & \\ \text { D } & & \sqrt{ } & \\ \text { D } & \sqrt{ } & \sqrt{ } & \end{array}$

$\sqrt{ }$ Gusakova, 1983

Zechmeister et al., 1932a

Zechmeister et al., 1932b

Banaszkiewicz et al., 1963

Goodwin, 1954

Toth \& Szabolics, 1981

Movchan, 1960

Baszynski, 1954

Fishman \& Chikovani, 1990a

Jui et al., 1998

Jui et al., 1998

Jui et al., 1998

Gaind et al., 1970

Laddha \& Jolly, 1985

$\sqrt{ }$ Bureau \& Bushway, 1986

Deli et al., 1996

Czinkotai et al., 1989

Biacs et al., 1993

Rodrigues et al.,1988

Mosquera et al., 1992

Mosquera et al., 1994

Mosquera et al., 1993

Gregory et al., 1987

Carcinus maenas

Portunidae

Carica papaya

Caricaceae

S

D

$\begin{array}{lll}\sqrt{ } & \sqrt{ } \\ \sqrt{ } & \end{array}$

Czeczuga, 1975

$\sqrt{ }$ Rao, 1967

Subbarayan \& Cama, 1964

Topuriya, 1990

Giri et al., 1980

Yamamoto, 1964

Strocchi et al., 1977

Baszynski, 1954

Getahun et al., 1973

Yadav et al., 1987

Czeczuga, 1988

Czeczuga, 1980a

Czeczuga, 1985b

Czeczuga \& Alstrup, 1987

Czeczuga \& Olech, 1990

Czeczuga \& Olech, 1990

Czeczuga \& Alstrup, 1987

Czeczuga \& Alstrup, 1987

Czeczuga \& Olech, 1990

Czeczuga \& Alstrup, 1987

Fiksdahl et al., 1984a

Kitayama \& Takakura, 1991

Francis et al., 1975

Burczyk, 1987

Burczyk, 1987

Burczyk, 1987

Burczyk, 1987

Takaichi et al., 1997

Takaichi et al., 1995

Pfeifhofer \& Grill, 1984

Ookubo \& Matsuno, 1985 


\begin{tabular}{|c|c|c|c|c|c|c|c|c|}
\hline \multirow[b]{2}{*}{ Species } & \multirow[b]{2}{*}{ Family } & \multirow[b]{2}{*}{ Group } & \multicolumn{5}{|c|}{ Carotene series } & \multirow[b]{2}{*}{ Reference } \\
\hline & & & $\alpha-C$ & $\beta-C$ & $\gamma-\mathrm{C}$ & $\delta-\mathrm{C}$ & $\varepsilon-\mathrm{C}$ & \\
\hline Citrus aurantium & Rutaceae & $\mathrm{D}$ & & $\sqrt{ }$ & & & & Toth \& Szabolics, 1981 \\
\hline Citrus limon & Rutaceae & $\mathrm{D}$ & $\sqrt{ }$ & $\sqrt{ }$ & & & & Kutateladze, 1974 \\
\hline Citrus paradisi & Rutaceae & $\mathrm{D}$ & $\sqrt{ }$ & $\sqrt{ }$ & $\sqrt{ }$ & & $\sqrt{ }$ & $\begin{array}{l}\text { Bureau \& Bushway, } 1986 \\
\text { Heinonen et al., } 1989 \\
\text { Rouseff et al., } 1992\end{array}$ \\
\hline Citrus sinensis & Rutaceae & $\mathrm{D}$ & $\sqrt{ }$ & $\sqrt{ }$ & & & & $\begin{array}{l}\text { Bureau \& Bushway, } 1986 \\
\text { Malachi et al., } 1974 \\
\text { Shim et al., } 1994 \\
\text { Kudritskaya et al., } 1983\end{array}$ \\
\hline Cladonia amaurocraea & Cladoniaceae & $\mathrm{L}$ & $\sqrt{ }$ & $\sqrt{ }$ & & & & Czeczuga \& Olech, 1990 \\
\hline Cladonia cenotea & Cladoniaceae & $\mathrm{L}$ & & $\sqrt{ }$ & & & & Czeczuga, 1985a \\
\hline Cladonia coccifera & Cladoniaceae & $\mathrm{L}$ & $\sqrt{ }$ & $\sqrt{ }$ & & & & $\begin{array}{l}\text { Czeczuga \& Olech, } 1990 \\
\text { Czeczuga, 1985a }\end{array}$ \\
\hline Cladonia cornuta & Cladoniaceae & $\mathrm{L}$ & $\sqrt{ }$ & $\sqrt{ }$ & & & & Czeczuga, 1985a \\
\hline Cladonia crispata & Cladoniaceae & $\mathrm{L}$ & & $\sqrt{ }$ & & & & Czeczuga, 1985a \\
\hline Cladonia deformis & Cladoniaceae & $\mathrm{L}$ & & $\sqrt{ }$ & & & & Czeczuga, 1985a \\
\hline Cladonia degenerans & Cladoniaceae & $\mathrm{L}$ & $\sqrt{ }$ & $\sqrt{ }$ & & & & Czeczuga, 1985a \\
\hline Cladonia foliacea & Cladoniaceae & $\mathrm{L}$ & & $\sqrt{ }$ & & & & Czeczuga et al., 1988 \\
\hline Cladonia furcata & Cladoniaceae & $\mathrm{L}$ & & $\sqrt{ }$ & & & & Czeczuga, 1985a \\
\hline Cladonia glauca & Cladoniaceae & $\mathrm{L}$ & & $\sqrt{ }$ & & & & Czeczuga, 1985a \\
\hline Cladonia gracilis & Cladoniaceae & $\mathrm{L}$ & & $\sqrt{ }$ & & & & Czeczuga, 1985a \\
\hline Cladonia impexa & Cladoniaceae & $\mathrm{L}$ & $\sqrt{ }$ & $\sqrt{ }$ & & & & Czeczuga, 1985a \\
\hline Cladonia macilenta & Cladoniaceae & $\mathrm{L}$ & $\sqrt{ }$ & & & & & Czeczuga, 1985a \\
\hline Cladonia mitis & Cladoniaceae & $\mathrm{L}$ & & $\sqrt{ }$ & & & & Czeczuga \& Alstrup, 1987 \\
\hline Cladonia nylanderi & Cladoniaceae & $\mathrm{L}$ & & $\sqrt{ }$ & & & & Czeczuga et al., 1988 \\
\hline Cladonia ochrochlora & Cladoniaceae & $\mathrm{L}$ & & $\sqrt{ }$ & & & & Czeczuga, 1985a \\
\hline Cladonia rangiferina & Cladoniaceae & $\mathrm{L}$ & & $\sqrt{ }$ & & & & Czeczuga, 1985a \\
\hline Cladonia scabriuscula & Cladoniaceae & $\mathrm{L}$ & & $\sqrt{ }$ & & & & Czeczuga, 1985a \\
\hline Cladonia sylvatica & Cladoniaceae & $\mathrm{L}$ & & $\sqrt{ }$ & & & & Czeczuga, 1985a \\
\hline Cladonia turgida & Cladoniaceae & $\mathrm{L}$ & & $\sqrt{ }$ & & & & Czeczuga, 1985a \\
\hline Cladonia verticillata & Cladoniaceae & $\mathrm{L}$ & & $\sqrt{ }$ & & & & Czeczuga, 1985a \\
\hline Cochlospermum vitifoleum & Cochlospermaceae & $\mathrm{D}$ & & $\sqrt{ }$ & $\sqrt{ }$ & & & Dixit \& Srivastava, 1992a \\
\hline Colocasia esculenta & Araceae & M & & $\sqrt{ }$ & & & & Rao, 1967 \\
\hline Coniogramme japonica & Pteridaceae & $\mathrm{FE}$ & $\sqrt{ }$ & & & & & Czeczuga, 1985b \\
\hline Corallina elongata & Corallinaceae & A & & $\sqrt{ }$ & & & & Palermo et al., 1991 \\
\hline Corallina officinalis & Corallinaceae & $\mathrm{A}$ & & $\sqrt{ }$ & & & & Palermo et al., 1991 \\
\hline Cornicularia divergens & Parmeliaceae & $\mathrm{L}$ & & $\sqrt{ }$ & & & & Czeczuga \& Alstrup, 1987 \\
\hline Crambe abyssinica & Brassicaceae & $\mathrm{D}$ & & $\sqrt{ }$ & & & & Baszynski, 1954 \\
\hline Crepis tectorum & Asteraceae & $\mathrm{D}$ & $\sqrt{ }$ & & $\sqrt{ }$ & & & Sergeeva et al., 1984 \\
\hline Crocus sativus & Iridaceae & M & & $\sqrt{ }$ & $\sqrt{ }$ & & & $\begin{array}{l}\text { Duquenois, } 1972 \\
\text { Kuhn \& Winterstein, } 1933 \\
\text { Pfander et al., } 1982\end{array}$ \\
\hline Ctenopharyngodon idella & Cyprinidae & $\mathrm{S}$ & & $\sqrt{ }$ & & & & Czeczuga, 1981 \\
\hline Cucumis melo & Cucurbitaceae & $\mathrm{D}$ & $\sqrt{ }$ & $\sqrt{ }$ & & & $\sqrt{ }$ & $\begin{array}{l}\text { Bureau \& Bushway, } 1986 \\
\text { Rojas \& Diaz, } 1977\end{array}$ \\
\hline Cucumis sativus & Cucurbitaceae & $\mathrm{D}$ & & $\sqrt{ }$ & & & & Granado et al., 1992 \\
\hline Cucurbita maxima & Cucurbitaceae & $\mathrm{D}$ & & $\sqrt{ }$ & & & & Neamtu et al., 1990 \\
\hline Cucurbita moschata & Cucurbitaceae & $\mathrm{D}$ & & $\sqrt{ }$ & & & & Doiiode \& Sulledmath, 1986 \\
\hline Cucurbita pepo & Cucurbitaceae & $\mathrm{D}$ & & $\sqrt{ }$ & & & & $\begin{array}{l}\text { Granado et al., } 1992 \\
\text { Neamtu et al., } 1990\end{array}$ \\
\hline Cucurbita $\mathrm{sp}$ & Cucurbitaceae & $\mathrm{D}$ & $\sqrt{ }$ & $\sqrt{ }$ & & & & Bureau \& Bushway, 1986 \\
\hline Cuscuta australis & Convolvulaceae & $\mathrm{D}$ & & $\sqrt{ }$ & $\sqrt{ }$ & & & Baccarini et al., 1965 \\
\hline Cyclopterus lumpus & Cyclopteridae & $\mathrm{S}$ & & $\sqrt{ }$ & & & & Czeczuga, 1982 \\
\hline Cynara scolymus & Asteraceae & $\mathrm{D}$ & & $\sqrt{ }$ & & & & Granado et al., 1992 \\
\hline Cyrtomium falcatum & Dryopteridaceae & FE & $\sqrt{ }$ & $\sqrt{ }$ & & & & Czeczuga, 1985b \\
\hline Cystoclonium purpureum & Rhodophyllidaceae & A & $\sqrt{ }$ & & & & & Findlay \& Patil, 1986 \\
\hline Cystopteris bulbifera & Dryopteridaceae & $\mathrm{FE}$ & & $\sqrt{ }$ & & & & Czeczuga, 1985b \\
\hline Cystopteris fragilis & Dryopteridaceae & FE & & $\sqrt{ }$ & & & & Czeczuga, $1985 b$ \\
\hline Dactylis glomerata & Poaceae & M & & $\sqrt{ }$ & & & & Pollard, 1936 \\
\hline Daucus carota & Apiaceae & $\mathrm{D}$ & $\sqrt{ }$ & $\sqrt{ }$ & $\sqrt{ }$ & & $\sqrt{ }$ & $\begin{array}{l}\text { Granado et al., } 1992 \\
\text { Rao, } 1967 \\
\text { Bureau \& Bushway, } 1986 \\
\text { Coxon et al., } 1973 \\
\text { Rhodes \& Hall, } 1975 \\
\text { Chandra \& Nair, } 1997 \\
\text { Barth et al., } 1995 \\
\text { Vega et al., } 1996\end{array}$ \\
\hline
\end{tabular}




\begin{tabular}{|c|c|c|c|c|c|c|c|c|}
\hline \multirow[b]{2}{*}{ Species } & \multirow[b]{2}{*}{ Family } & \multirow[b]{2}{*}{ Group } & \multicolumn{5}{|c|}{ Carotene series } & \multirow[b]{2}{*}{ Reference } \\
\hline & & & $\alpha-\mathrm{C}$ & $\beta-\mathrm{C}$ & $\gamma-\mathrm{C}$ & $\delta-\mathrm{C}$ & $\varepsilon-\mathrm{C}$ & \\
\hline & & & & & & & & $\begin{array}{l}\text { Van Breemen, } 1996 \\
\text { Sun, } 1994 \\
\text { Sato, } 1992 \\
\text { Mok et al., } 1976 \\
\text { Czygan \& Herboth, } 1976 \\
\text { Toth et al., } 1995 \\
\text { Koch et al, } 1991 \\
\text { Buishand \& Gabelman, } 1978 \\
\text { Baruffaldi et al., 1981 } \\
\text { Sarma \& Sarma, } 1978 \\
\text { Nishi et al., } 1974\end{array}$ \\
\hline Davallia solida & Davalliaceae & FE & $\sqrt{ }$ & & & & & Czeczuga, $1985 b$ \\
\hline Dermatocarpon miniatum & Verrucariaceae & $\mathrm{L}$ & & $\sqrt{ }$ & & & & Czeczuga \& Alstrup, 1987 \\
\hline Desmarestia aculeata & Desmarestiaceae & $\mathrm{A}$ & & $\sqrt{ }$ & & & & Findlay \& Patil, 1985 \\
\hline Didemnum moselyi & Didemnidae & $\mathrm{S}$ & & $\sqrt{ }$ & & & & Ookubo \& Matsuno, 1985 \\
\hline Dioscorea cayenensis & Dioscoreaceae & M & & $\sqrt{ }$ & & & & Martin \& Ruberte, 1975 \\
\hline Dioscorea versicolor & Dioscoreaceae & M & & $\sqrt{ }$ & & & $\sqrt{ }$ & Rao, 1967 \\
\hline Diospyros kaki & Ebenaceae & $\mathrm{D}$ & $\sqrt{ }$ & $\sqrt{ }$ & $\sqrt{ }$ & & $\sqrt{ }$ & $\begin{array}{l}\text { Fishman \& Chikovani, } \\
\text { 1990b } \\
\text { Kudritskaya et al., } 1984 \\
\text { Ebert \& Gross, } 1985\end{array}$ \\
\hline Diplazium sibiricum & Dryopteridaceae & FE & $\sqrt{ }$ & $\sqrt{ }$ & & & & Czeczuga, 1985b \\
\hline Drepanocladus fluitans & Amblystegiaceae & $\mathrm{FE}$ & & $\sqrt{ }$ & & & & Boston et al., 1991 \\
\hline Dryopteris carthusiana & Dryopteridaceae & FE & $\sqrt{ }$ & $\sqrt{ }$ & & & & Czeczuga, 1985b \\
\hline Dryopteris dilatata & Dryopteridaceae & $\mathrm{FE}$ & & $\sqrt{ }$ & & & & Czeczuga, 1985b \\
\hline Dryopteris filix-mas & Dryopteridaceae & FE & & $\sqrt{ }$ & & & & Czeczuga, $1985 b$ \\
\hline Dryopteris parasitica & Dryopteridaceae & $\mathrm{FE}$ & $\sqrt{ }$ & & & & & Czeczuga, 1985b \\
\hline Dunaliella bardawil & Dunaliellaceae & A & $\sqrt{ }$ & $\sqrt{ }$ & & & & $\begin{array}{l}\text { Ben-Amotz et al., } 1990 \\
\text { Takenaka et al., } 1993 \\
\text { Mokady et al., } 1989\end{array}$ \\
\hline Dunaliella salina & Dunaliellaceae & A & $\sqrt{ }$ & $\sqrt{ }$ & & & & $\begin{array}{l}\text { Ben-Amotz et al., } 1990 \\
\text { Chen et al., } 1995\end{array}$ \\
\hline Elaeis guineensis & Arecaceae & M & $\sqrt{ }$ & $\sqrt{ }$ & $\sqrt{ }$ & & & $\begin{array}{l}\text { Mariath et al., } 1989 \\
\text { Argoud, } 1958 \\
\text { Manorama \& Rukmini, } 1992\end{array}$ \\
\hline Eleutherococcus koreanus & Araliaceae & $\mathrm{D}$ & & $\sqrt{ }$ & & & & Bladt et al., 1990 \\
\hline Eleutherococcus senticosus & Araliaceae & $\mathrm{D}$ & & $\sqrt{ }$ & & & & Zorikov \& Burii, 1974 \\
\hline Equisetum arvensi & Equisetaceae & $\mathrm{FE}$ & $\sqrt{ }$ & $\sqrt{ }$ & & & & Czeczuga, $1985 b$ \\
\hline Equisetum fluviatile & Equisetaceae & FE & $\sqrt{ }$ & $\sqrt{ }$ & $\sqrt{ }$ & & & Czeczuga, $1985 b$ \\
\hline Equisetum hyemale & Equisetaceae & $\mathrm{FE}$ & $\sqrt{ }$ & $\sqrt{ }$ & & & & Czeczuga, 1985b \\
\hline Equisetum palustre & Equisetaceae & $\mathrm{FE}$ & & $\sqrt{ }$ & & & & Czeczuga, 1985b \\
\hline Equisetum pratense & Equisetaceae & $\mathrm{FE}$ & & $\sqrt{ }$ & & & & Czeczuga, 1985b \\
\hline Equisetum sylvaticum & Equisetaceae & FE & $\sqrt{ }$ & $\sqrt{ }$ & & & & Czeczuga, $1985 b$ \\
\hline Equisetum telmateia & Equisetaceae & FE & $\sqrt{ }$ & $\sqrt{ }$ & & & & Czeczuga, $1985 b$ \\
\hline Eriobotrya japonica & Rosaceae & $\mathrm{D}$ & $\sqrt{ }$ & & & & & Ben-Amotz et al., 1990 \\
\hline Erysimum diffusum & Brassicaceae & $\mathrm{D}$ & $\sqrt{ }$ & & & & & Gusakova, 1983 \\
\hline Erythrotrichia carnea & Erythropeltidaceae & A & & $\sqrt{ }$ & & & & Bjoernland, 1984 \\
\hline Euglena gracilis & Euglenaceae & A & & $\sqrt{ }$ & & & $\sqrt{ }$ & Gross et al., 1975 \\
\hline Euglena sanguinea & Euglenaceae & $\mathrm{A}$ & & $\sqrt{ }$ & & & & Grung \& Jensen, 1993 \\
\hline Fibrocapsa japonica & To be determined & A & & $\sqrt{ }$ & & & & Fiksdahl et al., 1984a \\
\hline Ficus carica & Moraceae & $\mathrm{D}$ & & $\sqrt{ }$ & & & & Karag'ozova, 1974 \\
\hline Fontinalis antipyretica & Fontinalaceae & $\mathrm{FE}$ & $\sqrt{ }$ & $\sqrt{ }$ & & & & $\begin{array}{l}\text { Czeczuga, 1980a } \\
\text { Boston et al., } 1991\end{array}$ \\
\hline Fontinalis squamosa & Fontinalaceae & FE & $\sqrt{ }$ & $\sqrt{ }$ & & & & Czeczuga, 1980a \\
\hline Fragaria ananassa & Rosaceae & $\mathrm{D}$ & & $\sqrt{ }$ & & & & Gross, 1972 \\
\hline Fragaria $\mathrm{sp}$ & Rosaceae & $\mathrm{D}$ & $\sqrt{ }$ & $\sqrt{ }$ & & & & Bureau \& Bushway, 1986 \\
\hline Fucus serratus & Fucaceae & $\mathrm{A}$ & & $\sqrt{ }$ & & & & Haugan \& Jensen, 1954 \\
\hline Fucus vesiculosus & Fucaceae & A & & $\sqrt{ }$ & & & & $\begin{array}{l}\text { Haugan \& Jensen, } 1954 \\
\text { Liaaen \& Sorensen, } 1955 \\
\text { Halsall \& Hills, } 1971\end{array}$ \\
\hline Funaria hygrometrica & Funariaceae & $\mathrm{FE}$ & & $\sqrt{ }$ & $\sqrt{ }$ & & & Czeczuga, 1980a \\
\hline Fusinus perplexus & Fasciolariidae & $\mathrm{S}$ & & $\sqrt{ }$ & & & & Matsuno \& Tsushima, 1989 \\
\hline Galium helderichii & Rubiaceae & $\mathrm{D}$ & & $\sqrt{ }$ & & & & Tzakou et al., 1988 \\
\hline Galium mollugo & Rubiaceae & $\mathrm{D}$ & & $\sqrt{ }$ & & & & Shapiro et al., 1985 \\
\hline Galium tinctorium & Rubiaceae & $\mathrm{D}$ & & $\sqrt{ }$ & & & & Shapiro et al., 1985 \\
\hline Genista dentata & Fabaceae & $\mathrm{D}$ & $\sqrt{ }$ & $\sqrt{ }$ & & & & Schon \& Mesqita, 1936 \\
\hline Ginkgo biloba & Ginkgoaceae & G & $\sqrt{ }$ & $\sqrt{ }$ & $\sqrt{ }$ & & & $\begin{array}{l}\text { Yadav et al., } 1987 \\
\text { Matile et al., } 1992\end{array}$ \\
\hline
\end{tabular}




\begin{tabular}{|c|c|c|c|c|c|c|c|c|}
\hline \multirow[b]{2}{*}{ Species } & \multirow[b]{2}{*}{ Family } & \multirow[b]{2}{*}{ Group } & \multicolumn{5}{|c|}{ Carotene series } & \multirow[b]{2}{*}{ Reference } \\
\hline & & & $\alpha-\mathrm{C}$ & $\beta-\mathrm{C}$ & $\gamma-\mathrm{C}$ & $\delta-\mathrm{C}$ & $\varepsilon-\mathrm{C}$ & \\
\hline Glycine max & Fabaceae & $\mathrm{D}$ & & $\sqrt{ }$ & & & & $\begin{array}{l}\text { Rao, } 1967 \\
\text { Baszynski, } 1954\end{array}$ \\
\hline Gnetum africanum & Gnetaceae & $\mathrm{G}$ & & $\sqrt{ }$ & & & & Njoku et al., 1997 \\
\hline Gomphosphaeria aponina & Chroococaceae & A & & $\sqrt{ }$ & & & & $\begin{array}{l}\text { Eng-Wilmont \& Martin, } \\
1979\end{array}$ \\
\hline Gonyostomum semen & Vacuolariaceae & A & & $\sqrt{ }$ & & & & Fiksdahl et al., 1984a \\
\hline Gossypium barbadense & Malvaceae & $\mathrm{D}$ & & $\sqrt{ }$ & & & & Khodzhaev, 1981 \\
\hline Gossypium hirsutum & Malvaceae & $\mathrm{D}$ & $\sqrt{ }$ & $\sqrt{ }$ & & & & $\begin{array}{l}\text { Jones, } 1979 \\
\text { Hanny \& Hedin, } 1972\end{array}$ \\
\hline Gossypium sp & Malvaceae & $\mathrm{D}$ & $\sqrt{ }$ & $\sqrt{ }$ & & & & Mc Cormick, 1982 \\
\hline Gracilaria lichenoides & Gracilariaceae & A & & $\sqrt{ }$ & & & & Aihara \& Yamamoto, 1968 \\
\hline Grammatophora oceanica & Bacillariophyceae & A & & $\sqrt{ }$ & & & & Pennington et al., 1988 \\
\hline Grimmia pulvinata & Grimmiaceae & $\mathrm{FE}$ & & $\sqrt{ }$ & & & & Czeczuga, 1980a \\
\hline Gymnodinium catenatum & Gymnodiniaceae & A & & $\sqrt{ }$ & & & & Hallegraeff et al., 1991 \\
\hline Gymnogramma sulphurea & Pteridaceae & $\mathrm{FE}$ & $\sqrt{ }$ & $\sqrt{ }$ & & & & Czeczuga, 1985b \\
\hline Gyrodinium aureolum & Gymnodiniaceae & $\mathrm{S}$ & & $\sqrt{ }$ & & & & Tangen \& Bjoernland, 1981 \\
\hline Gyrodinium sp & Gymnodiniaceae & $\mathrm{S}$ & & $\sqrt{ }$ & & & & Bjoerland \& Tangen, 1979 \\
\hline Haematococcus lacustris & Haematococcaceae & $\mathrm{S}$ & & $\sqrt{ }$ & & & & Donkin, 1976 \\
\hline Halobacterium cutirubrum & Halobacteriaceae & $\mathrm{B}$ & & $\sqrt{ }$ & & & & Kushwaha et al., 1975 \\
\hline Halocynthia roretzi & Pyuridae & $\mathrm{S}$ & & $\sqrt{ }$ & & & & $\begin{array}{l}\text { Ookubo \& Matsuno, } 1985 \\
\text { Matsuno et al., 1984b }\end{array}$ \\
\hline Hedysarum polybotrys & Fabaceae & $\mathrm{D}$ & & $\sqrt{ }$ & & & & Jiang, 1989 \\
\hline Helianthemum numularum & Cistaceae & $\mathrm{D}$ & $\sqrt{ }$ & $\sqrt{ }$ & & & & Marius et al., 1994 \\
\hline Helianthus annuus & Asteraceae & $\mathrm{D}$ & & $\sqrt{ }$ & & & & Baszynski, 1954 \\
\hline Heliopsis scabra & Asteraceae & $\mathrm{D}$ & & $\sqrt{ }$ & & & & Raszeja \& Muszynska, 1980 \\
\hline Heterosigma akashiwo & Dinophyceae & A & & $\sqrt{ }$ & & & & Fiksdahl et al., $1984 \mathrm{~b}$ \\
\hline Hexabranchus sp & Hexabranchidae & $\mathrm{S}$ & & $\sqrt{ }$ & & & & Tanaka et al., 1992 \\
\hline Hibiscus esculentus & Malvaceae & $\mathrm{D}$ & $\sqrt{ }$ & $\sqrt{ }$ & & & & Bureau \& Bushway, 1986 \\
\hline Hibiscus sabdariffa & Malvaceae & $\mathrm{D}$ & & $\sqrt{ }$ & & & & Rao, 1967 \\
\hline Hibiscus syriacus & Malvaceae & $\mathrm{D}$ & $\sqrt{ }$ & $\sqrt{ }$ & & & & $\begin{array}{l}\text { Hanny \& Hedin, } 1972 \\
\text { Hanny et al., } 1972 \\
\text { Chernenko \& Umarov, } 1974\end{array}$ \\
\hline Hippophae rhamnoides & Elaeagnaceae & $\mathrm{D}$ & $\sqrt{ }$ & $\sqrt{ }$ & $\sqrt{ }$ & & & $\begin{array}{l}\text { Zorikov \& Burii, } 1974 \\
\text { Sergeeva et al., } 1979 \\
\text { Gachechiladze et al., } 1981 \\
\text { Lagazidze et al., } 1984 \\
\text { Zhmyrko et al., } 1978\end{array}$ \\
\hline Hydrilla verticillata & Hydrocharitaceae & M & & $\sqrt{ }$ & & & & Lin et al., 1979 \\
\hline Hylocomium splendens & Hylocomiaceae & $\mathrm{FE}$ & & $\sqrt{ }$ & & & & Czeczuga, 1980a \\
\hline Hypericum perforatum & Clusiaceae & $\mathrm{D}$ & $\sqrt{ }$ & $\sqrt{ }$ & & & & $\begin{array}{l}\text { Omarova et al., } 1997 \\
\text { Chaplinskaya, } 1956\end{array}$ \\
\hline Hypnum cupressiforme & Hypnaceae & $\mathrm{FE}$ & & $\sqrt{ }$ & $\sqrt{ }$ & & & Czeczuga, 1980a \\
\hline Ianthella basta & Ianthellidae & $\mathrm{S}$ & & $\sqrt{ }$ & & & & Ramdahl et al., 1981 \\
\hline Ianthella flabelliformis & Ianthellidae & $\mathrm{S}$ & & $\sqrt{ }$ & & & & Hertzberg et al., 1989 \\
\hline Ipomoea aquatica & Convolvulaceae & $\mathrm{D}$ & & $\sqrt{ }$ & & & & $\begin{array}{l}\text { Ortaliza et al., } 1969 \\
\text { Kasemsri et al., } 1952\end{array}$ \\
\hline Ipomoea batatas & Convolvulaceae & $\mathrm{D}$ & $\sqrt{ }$ & $\sqrt{ }$ & & & & $\begin{array}{l}\text { Bureau \& Bushway, } 1986 \\
\text { Ortaliza et al., } 1969\end{array}$ \\
\hline Iris germanica & Iridaceae & $\mathrm{M}$ & & $\sqrt{ }$ & & & & Buchecker \& Jensen, 1975 \\
\hline Iris pseudacorus & Iridaceae & $\mathrm{M}$ & & $\sqrt{ }$ & & & & O'Connor \& Drumm, 1941 \\
\hline Jania $\mathrm{sp}$ & Corallinaceae & A & & $\sqrt{ }$ & & & & Palermo et al., 1991 \\
\hline Lactuca sativa & Asteraceae & $\mathrm{D}$ & $\sqrt{ }$ & $\sqrt{ }$ & & & & $\begin{array}{l}\text { Granado et al., } 1992 \\
\text { Bureau \& Bushway, } 1986\end{array}$ \\
\hline Laminaria digitata & Laminariaceae & A & & $\sqrt{ }$ & & & & Haugan \& Jensen, 1994 \\
\hline Laminaria saccharina & Laminariaceae & A & & $\sqrt{ }$ & & & & Haugan \& Jensen, 1994 \\
\hline Leptura rubra & Cerambycidae & I & & $\sqrt{ }$ & & & & Czeczuga, 1988 \\
\hline Lespedeza sp & Fabaceae & $\mathrm{D}$ & & $\sqrt{ }$ & & & & Zorikov \& Burii, 1974 \\
\hline Leucobryum glaucum & Leucobryaceae & $\mathrm{FE}$ & $\sqrt{ }$ & $\sqrt{ }$ & & & & Czeczuga, 1980a \\
\hline Ligia exotica & Ligiidae & $\mathrm{S}$ & & $\sqrt{ }$ & & & & Matsuno et al., 1990 \\
\hline Lilium tenuifolium & Liliaceae & M & & $\sqrt{ }$ & & & & Partali et al., 1987 \\
\hline Lobaria linita & Lobariaceae & $\mathrm{L}$ & $\sqrt{ }$ & $\sqrt{ }$ & & & & Czeczuga \& Olech, 1990 \\
\hline Lolium perenne & Poaceae & $\mathrm{M}$ & & $\sqrt{ }$ & & & & Tamas \& Popescu, 1985 \\
\hline Lonicera altaica & Caprifoliaceae & $\mathrm{D}$ & & $\sqrt{ }$ & & & & Shapiro et al., 1981 \\
\hline Lonicera caerulea & Caprifoliaceae & $\mathrm{D}$ & & $\sqrt{ }$ & & & & Shapiro et al., 1981 \\
\hline Lonicera edulis & Caprifoliaceae & $\mathrm{D}$ & & $\sqrt{ }$ & & & & Shapiro et al., 1981 \\
\hline Lonicera japonica & Caprifoliaceae & $\mathrm{D}$ & & $\sqrt{ }$ & $\sqrt{ }$ & & $\sqrt{ }$ & Goodwin, 1952 \\
\hline Lota lota & Gadidae & $\mathrm{S}$ & & $\sqrt{ }$ & & & & Czeczuga, 1978 \\
\hline Lotus corniculatus & Fabaceae & $\mathrm{D}$ & & $\sqrt{ }$ & & & & Ceruti et al., 1972 \\
\hline
\end{tabular}




\begin{tabular}{|c|c|c|c|c|c|c|c|c|}
\hline \multirow[b]{2}{*}{ Species } & \multirow[b]{2}{*}{ Family } & \multirow[b]{2}{*}{ Group } & \multicolumn{5}{|c|}{ Carotene series } & \multirow[b]{2}{*}{ Reference } \\
\hline & & & $\alpha-\mathrm{C}$ & $\beta-\mathrm{C}$ & $\gamma-\mathrm{C}$ & $\delta-\mathrm{C}$ & $\varepsilon-\mathrm{C}$ & \\
\hline Lupinus albus & Fabaceae & $\mathrm{D}$ & & $\sqrt{ }$ & & & & El-Fifrawi \& Hudson, 1979 \\
\hline Lupinus angustifolius & Fabaceae & $\mathrm{D}$ & & $\sqrt{ }$ & & & & El-Fifrawi \& Hudson, 1979 \\
\hline Lupinus luteus & Fabaceae & $\mathrm{D}$ & & $\sqrt{ }$ & & & & El-Fifrawi \& Hudson, 1979 \\
\hline Lupinus mutabilis & Fabaceae & $\mathrm{D}$ & & $\sqrt{ }$ & & & & El-Fifrawi \& Hudson, 1979 \\
\hline Lycopersicon cheesmanii & Solanaceae & $\mathrm{D}$ & & $\sqrt{ }$ & & & & Chalukova et al., 1985 \\
\hline Lycopersicon esculentum & Solanaceae & $\mathrm{D}$ & & $\sqrt{ }$ & $\sqrt{ }$ & & & $\begin{array}{l}\text { Granado et al., } 1992 \\
\text { Parry \& Horgan, } 1992 \\
\text { Laval-Martin et al., } 1975\end{array}$ \\
\hline Lycopodium annotinum & Lycopodiaceae & FE & & $\sqrt{ }$ & & & & Czeczuga, $1985 b$ \\
\hline Lycopodium clavatum & Lycopodiaceae & FE & & $\sqrt{ }$ & & & & Czeczuga, $1985 b$ \\
\hline Lycopodium complanatum & Lycopodiaceae & FE & & $\sqrt{ }$ & & & & Czeczuga, 1985 \\
\hline Lycopodium selago & Lycopodiaceae & FE & & $\sqrt{ }$ & & & & Czeczuga, $1985 b$ \\
\hline Malpighia punicifolia & Malpighiaceae & $\mathrm{D}$ & & $\sqrt{ }$ & $\sqrt{ }$ & & $\sqrt{ }$ & Baraud, 1958 \\
\hline Mangifera indica & Anacardiaceae & $\mathrm{D}$ & $\sqrt{ }$ & $\sqrt{ }$ & $\sqrt{ }$ & & & $\begin{array}{l}\text { Meimban et al., } 1983 \\
\text { Mercadante et al., } 1988 \text { b }\end{array}$ \\
\hline Mantoniella squamata & Nephroselmidaceae & A & & $\sqrt{ }$ & & & & Egeland et al., 1995 \\
\hline Marchantia polymorpha & Marchantiaceae & FE & $\sqrt{ }$ & $\sqrt{ }$ & $\sqrt{ }$ & & & Czeczuga, 1980a \\
\hline Matteuccia struthiopteris & Dryopteridaceae & $\mathrm{FE}$ & $\sqrt{ }$ & $\sqrt{ }$ & & & & $\begin{array}{l}\text { Czeczuga, 1985b } \\
\text { Bushway et al., } 1985\end{array}$ \\
\hline Mauritia vinifera & Arecaceae & M & & $\sqrt{ }$ & & & & Mariath et al., 1989 \\
\hline Medicago arborea & Fabaceae & $\mathrm{D}$ & & $\sqrt{ }$ & & & & Ignasiak \& Lesins, 1975 \\
\hline Medicago cancellata & Fabaceae & $\mathrm{D}$ & & $\sqrt{ }$ & & & & Ignasiak \& Lesins, 1975 \\
\hline Medicago carstiensis & Fabaceae & $\mathrm{D}$ & $\sqrt{ }$ & $\sqrt{ }$ & & & & Ignasiak \& Lesins, 1975 \\
\hline Medicago cretacea & Fabaceae & $\mathrm{D}$ & & $\sqrt{ }$ & & & & Ignasiak \& Lesins, 1975 \\
\hline Medicago daghestanica & Fabaceae & $\mathrm{D}$ & $\sqrt{ }$ & $\sqrt{ }$ & & & & Ignasiak \& Lesins, 1975 \\
\hline Medicago falcata & Fabaceae & $\mathrm{D}$ & & $\sqrt{ }$ & & & & Ignasiak \& Lesins, 1975 \\
\hline Medicago glomerata & Fabaceae & $\mathrm{D}$ & & $\sqrt{ }$ & & & & Ignasiak \& Lesins, 1975 \\
\hline Medicago glutinosa & Fabaceae & $\mathrm{D}$ & & $\sqrt{ }$ & & & & Ignasiak \& Lesins, 1975 \\
\hline Medicago hemicycla & Fabaceae & $\mathrm{D}$ & $\sqrt{ }$ & $\sqrt{ }$ & & & & Ignasiak \& Lesins, 1975 \\
\hline Medicago hybrida & Fabaceae & $\mathrm{D}$ & $\sqrt{ }$ & $\sqrt{ }$ & & & & Ignasiak \& Lesins, 1975 \\
\hline Medicago leiocarpa & Fabaceae & $\mathrm{D}$ & $\sqrt{ }$ & $\sqrt{ }$ & & & & Ignasiak \& Lesins, 1975 \\
\hline Medicago marina & Fabaceae & $\mathrm{D}$ & $\sqrt{ }$ & $\sqrt{ }$ & & & & Ignasiak \& Lesins, 1975 \\
\hline Medicago papillosa & Fabaceae & $\mathrm{D}$ & & $\sqrt{ }$ & & & & Ignasiak \& Lesins, 1975 \\
\hline Medicago pironae & Fabaceae & $\mathrm{D}$ & $\sqrt{ }$ & $\sqrt{ }$ & & & & Ignasiak \& Lesins, 1975 \\
\hline Medicago platycarpos & Fabaceae & $\mathrm{D}$ & $\sqrt{ }$ & $\sqrt{ }$ & & & & Ignasiak \& Lesins, 1975 \\
\hline Medicago prostrata & Fabaceae & $\mathrm{D}$ & & $\sqrt{ }$ & & & & Ignasiak \& Lesins, 1975 \\
\hline Medicago rhodopea & Fabaceae & $\mathrm{D}$ & $\sqrt{ }$ & $\sqrt{ }$ & & & & Ignasiak \& Lesins, 1975 \\
\hline Medicago rupestris & Fabaceae & $\mathrm{D}$ & & $\sqrt{ }$ & & & & Ignasiak \& Lesins, 1975 \\
\hline Medicago ruthenica & Fabaceae & $\mathrm{D}$ & $\sqrt{ }$ & $\sqrt{ }$ & & & & Ignasiak \& Lesins, 1975 \\
\hline Medicago sativa & Fabaceae & $\mathrm{D}$ & & $\sqrt{ }$ & & & & $\begin{array}{l}\text { Toth et al., } 1995 \\
\text { Ignasiak \& Lesins, } 1975\end{array}$ \\
\hline Medicago saxatilis & Fabaceae & $\mathrm{D}$ & $\sqrt{ }$ & $\sqrt{ }$ & & & & Ignasiak \& Lesins, 1975 \\
\hline Medicago suffruticosa & Fabaceae & $\mathrm{D}$ & $\sqrt{ }$ & $\sqrt{ }$ & & & & Ignasiak \& Lesins, 1975 \\
\hline Melinis minutiflora & Poaceae & $\mathrm{M}$ & & $\sqrt{ }$ & & & & Calle Alvarez et al., 1986 \\
\hline Metasequoia glyptosboides & Taxodiaceae & $\mathrm{G}$ & $\sqrt{ }$ & $\sqrt{ }$ & $\sqrt{ }$ & & & Czeczuga, 1987 \\
\hline Microlepia speluncae & Dennstaedtiaceae & $\mathrm{FE}$ & $\sqrt{ }$ & $\sqrt{ }$ & & & & Czeczuga, 1985b \\
\hline Millingtonia hortensis & Bignoniaceae & $\mathrm{D}$ & & $\sqrt{ }$ & & & & Mangayarkarasi et al.,1984 \\
\hline Mimosa biuncifera & Fabaceae & $\mathrm{D}$ & & $\sqrt{ }$ & & & & Kudritskaya et al., 1988a \\
\hline Mimulus cupreus & Scrophulariaceae & $\mathrm{D}$ & & $\sqrt{ }$ & & & & Goodwin \& Thomas, 1964 \\
\hline Mimulus tigrinus & Scrophulariaceae & $\mathrm{D}$ & & $\sqrt{ }$ & & & & Goodwin \& Thomas, 1964 \\
\hline Mnium affine & Mniaceae & FE & $\sqrt{ }$ & $\sqrt{ }$ & & & & Czeczuga, 1980a \\
\hline Mnium cuspidatum & Mniaceae & FE & $\sqrt{ }$ & $\sqrt{ }$ & & & & Czeczuga, 1980a \\
\hline Mnium undulatum & Mniaceae & FE & $\sqrt{ }$ & $\sqrt{ }$ & & & & Czeczuga, 1980a \\
\hline Momordica charantia & Cucurbitaceae & $\mathrm{D}$ & & $\sqrt{ }$ & $\sqrt{ }$ & $\sqrt{ }$ & & Rodriguez et al., 1976 \\
\hline Monarda didyma & Lamiaceae & $\mathrm{D}$ & & $\sqrt{ }$ & & & & Brieskorn \& Meister, 1965 \\
\hline Monochamus pistor & Cerambycidae & I & & $\sqrt{ }$ & & & & Czeczuga, 1988 \\
\hline Montanoa karwinski & Asteraceae & $\mathrm{D}$ & & $\sqrt{ }$ & & & & Quijano et al., 1995 \\
\hline Morinda citrifolia & Rubiaceae & $\mathrm{D}$ & & $\sqrt{ }$ & & & & Aalbersberg et al., 1993 \\
\hline Moringa pterygosperma & Moringaceae & $\mathrm{D}$ & & $\sqrt{ }$ & & & & Ortaliza et al., 1969 \\
\hline Morus alba & Moraceae & $\mathrm{D}$ & & $\sqrt{ }$ & & & & Yen et al., 1996 \\
\hline Murraya euchrestifolia & Rutaceae & $\mathrm{D}$ & & $\sqrt{ }$ & & & & $\mathrm{Wu}, 1991$ \\
\hline Mycobacterium brevicale & Mycobacteriaceae & A & $\sqrt{ }$ & $\sqrt{ }$ & & & & Daraseliya et al., 1979 \\
\hline Mycobacterium kansasii & Mycobacteriaceae & A & & $\sqrt{ }$ & & & & David, 1974 \\
\hline Navicula delognei & Naviculaceae & A & & $\sqrt{ }$ & & & & Findlay \& Patil, 1984 \\
\hline Navicula pelliculosa & Naviculaceae & A & & $\sqrt{ }$ & & & & Pennington et al., 1988 \\
\hline Nephrolepis exaltata & Nephrolepidaceae & FE & & $\sqrt{ }$ & & & & Czeczuga, $1985 \mathrm{~b}$ \\
\hline Nephroma arcticum & Peltigeraceae & $\mathrm{L}$ & & $\sqrt{ }$ & & & & Czeczuga \& Olech, 1990. \\
\hline
\end{tabular}




\begin{tabular}{|c|c|c|c|c|c|c|c|c|}
\hline \multirow[b]{2}{*}{ Species } & \multirow[b]{2}{*}{ Family } & \multirow[b]{2}{*}{ Group } & \multicolumn{5}{|c|}{ Carotene series } & \multirow[b]{2}{*}{ Reference } \\
\hline & & & $\alpha-C$ & $\beta-\mathrm{C}$ & $\gamma-\mathrm{C}$ & $\delta-\mathrm{C}$ & $\varepsilon-\mathrm{C}$ & \\
\hline Nephroma laevigatum & Peltigeraceae & $\mathrm{L}$ & & $\sqrt{ }$ & & & & Czeczuga et al., 1988 \\
\hline Nephroma parile & Peltigeraceae & $\mathrm{L}$ & & $\sqrt{ }$ & & & & Czeczuga \& Alstrup, 1987 \\
\hline Nicotiana plumbaginifolia & Solanaceae & $\mathrm{D}$ & & $\sqrt{ }$ & & & & Parry \& Horgan, 1992 \\
\hline Nicotiana tabacum & Solanaceae & $\mathrm{D}$ & & $\sqrt{ }$ & & & & Lichtenthaler et al., 1975 \\
\hline Nitzschia $\mathrm{sp}$ & Nitzschiaceae & $\mathrm{A}$ & & $\sqrt{ }$ & & & & Pennington et al., 1988 \\
\hline Ocimum sanctum & Lamiaceae & $\mathrm{D}$ & & $\sqrt{ }$ & & & & Skaltsa et al., 1987 \\
\hline Odontoschisma denudatum & Odontoschismaceae & $\mathrm{FE}$ & $\sqrt{ }$ & $\sqrt{ }$ & & & & Czeczuga, 1980a \\
\hline Olea europaea & Oleaceae & $\mathrm{D}$ & $\sqrt{ }$ & $\sqrt{ }$ & $\sqrt{ }$ & & & Golubev et al., 1986 \\
\hline Onoclea sensibilis & Dryopteridaceae & $\mathrm{FE}$ & & $\sqrt{ }$ & & & & Czeczuga,1985b \\
\hline Orthosiphon grandiflorus & Lamiaceae & $\mathrm{D}$ & $\sqrt{ }$ & $\sqrt{ }$ & & & & Kudritskaya et al., 1988b \\
\hline Orychophragmus violaceus & Brassicaceae & $\mathrm{D}$ & & $\sqrt{ }$ & & & & Li et al., 1997 \\
\hline Oscillatoria agardhii & Oscillatoriaceae & A & & $\sqrt{ }$ & & & & $\begin{array}{l}\text { Ronneberg et al., } 1980 \\
\text { Foss et al., } 1986\end{array}$ \\
\hline Oscillatoria bornetii & Oscillatoriaceae & A & & $\sqrt{ }$ & & & & Foss et al., 1986 \\
\hline Oscillatoria limosa & Oscillatoriaceae & A & & $\sqrt{ }$ & & & & $\begin{array}{l}\text { Foss et al., } 1986 \\
\text { Francis et al., } 1970\end{array}$ \\
\hline Oscillatoria rubescens & Oscillatoriaceae & A & & $\sqrt{ }$ & & & & Ronneberg et al., 1980 \\
\hline Paralithodes brevipes & Lithodidae & $\mathrm{S}$ & & $\sqrt{ }$ & & & & Matsuno Maoka, 1988 \\
\hline Parmelia acetabulum & Parmeliaceae & $\mathrm{L}$ & & $\sqrt{ }$ & & & & Czeczuga et al., 1989 \\
\hline Passiflora edulis & Passifloraceae & $\mathrm{D}$ & & $\sqrt{ }$ & & & $\sqrt{ }$ & Mercadante et al., 1998a \\
\hline Pellaea rotundifolia & Pteridaceae & FE & & $\sqrt{ }$ & & & & Czeczuga, 1985b \\
\hline Pellia endiviaefolia & Pelliaceae & $\mathrm{FE}$ & & $\sqrt{ }$ & & & & Asakawa \& Takemoto, 1978 \\
\hline Pellia fabbroniana & Pelliaceae & $\mathrm{FE}$ & & $\sqrt{ }$ & & & & Czeczuga, 1980a \\
\hline Peltigera aphthosa & Peltigeraceae & $\mathrm{L}$ & $\sqrt{ }$ & $\sqrt{ }$ & & & & $\begin{array}{l}\text { Czeczuga, } 1986 \\
\text { Czeczuga, } 1980 \mathrm{~b}\end{array}$ \\
\hline Peltigera canina & Peltigeraceae & $\mathrm{L}$ & & $\sqrt{ }$ & & & & Czeczuga, 1980b \\
\hline Peltigera erumphens & Peltigeraceae & $\mathrm{L}$ & & $\sqrt{ }$ & & & & Czeczuga, 1980b \\
\hline Peltigera hazszlynskyi & Peltigeraceae & $\mathrm{L}$ & & $\sqrt{ }$ & & & $\sqrt{ }$ & Czeczuga, 1980b \\
\hline Peltigera leucophlebia & Peltigeraceae & $\mathrm{L}$ & & $\sqrt{ }$ & & & & $\begin{array}{l}\text { Czeczuga \& Olech, } 1990 \\
\text { Czeczuga, 1980b }\end{array}$ \\
\hline Peltigera malacea & Peltigeraceae & $\mathrm{L}$ & $\sqrt{ }$ & $\sqrt{ }$ & & & & $\begin{array}{l}\text { Czeczuga \& Olech, } 1990 \\
\text { Czeczuga, 1980b }\end{array}$ \\
\hline Peltigera polydactyla & Peltigeraceae & $\mathrm{L}$ & & $\sqrt{ }$ & & & & Czeczuga, 1980b \\
\hline Peltigera ponojensis & Peltigeraceae & $\mathrm{L}$ & & $\sqrt{ }$ & & & & Czeczuga et al., 1989 \\
\hline Peltigera praetexta & Peltigeraceae & $\mathrm{L}$ & $\sqrt{ }$ & $\sqrt{ }$ & & & $\sqrt{ }$ & Czeczuga, 1980b \\
\hline Peltigera rufescens & Peltigeraceae & $\mathrm{L}$ & & $\sqrt{ }$ & & & & $\begin{array}{l}\text { Czeczuga \& Alstrup, } 1987 \\
\text { Czeczuga, 1980b }\end{array}$ \\
\hline Pelvetia canaliculata & Fucaceae & A & & $\sqrt{ }$ & & & & Haugan \& Jensen, 1954 \\
\hline Penaeus japonicus & Penaeidae & $\mathrm{S}$ & & $\sqrt{ }$ & & & & Katagiri et al., 1987 \\
\hline Persea americana & Lauraceae & $\mathrm{D}$ & $\sqrt{ }$ & $\sqrt{ }$ & & & $\sqrt{ }$ & Gross et al., 1972 \\
\hline Petroselinum crispum & Apiaceae & $\mathrm{D}$ & & $\sqrt{ }$ & & & & Francis \& Isaken, 1989 \\
\hline Phaeocystis sp & Chrysocapsaceae & $\mathrm{S}$ & & $\sqrt{ }$ & & & & Bjornland et al., 1988 \\
\hline Phaffia rhodozyma & Hyphomycetes & FU & & $\sqrt{ }$ & & & & Yajima et al., 1993 \\
\hline Phaseolus vulgaris & Fabaceae & $\mathrm{D}$ & $\sqrt{ }$ & $\sqrt{ }$ & & & & $\begin{array}{l}\text { Granado et al., } 1992 \\
\text { Rao, } 1967 \\
\text { Parry \& Horgan, } 1992 \\
\text { Bureau \& Bushway, } 1986 \\
\text { Wu et al., } 1992 \\
\text { Lopez-Hernandez et al., } \\
1993\end{array}$ \\
\hline Phegopteris dryopteris & Thelypteridaceae & $\mathrm{FE}$ & & $\sqrt{ }$ & & & & Czeczuga, 1985b \\
\hline Pheodactylum tricornutum & To be determined & $\mathrm{A}$ & & $\sqrt{ }$ & & & & Pennington et al., 1988 \\
\hline Phormidium laminosum & Oscillatoriaceae & $\mathrm{A}$ & & $\sqrt{ }$ & & & & Fresnedo et al., 1991 \\
\hline Phycomyces blakesleeanus & Mucoraceae & FU & & $\sqrt{ }$ & $\sqrt{ }$ & & & $\begin{array}{l}\text { Bramley \& Davies, } 1975 \\
\text { Bramley \& Davies, } 1976 \\
\text { Elahi et al., } 1975 \\
\text { Eslava et al., } 1974 \\
\text { Desai et al., } 1975 \\
\text { Lee et al., } 1975\end{array}$ \\
\hline Physalis alkekengi & Solanaceae & $\mathrm{D}$ & & $\sqrt{ }$ & & & $\sqrt{ }$ & Baraud, 1958 \\
\hline Physconia pulverulacea & Physciaceae & $\mathrm{L}$ & $\sqrt{ }$ & $\sqrt{ }$ & & & & Czeczuga, 1986 \\
\hline Picea abies & Pinaceae & G & $\sqrt{ }$ & $\sqrt{ }$ & $\sqrt{ }$ & & & $\begin{array}{l}\text { Pfeifhofer \& Grill, } 1984 \\
\text { Grill et al., } 1984 \\
\text { Hoque, } 1988\end{array}$ \\
\hline Pinus massoniana & Pinaceae & G & & $\sqrt{ }$ & & & & Zhou et al., 1984 \\
\hline Pinus roxburghii & Pinaceae & G & & $\sqrt{ }$ & & & & Yadav et al., 1987 \\
\hline Piper betle & Piperaceae & $\mathrm{D}$ & & $\sqrt{ }$ & & & & Azuine et al., 1991 \\
\hline
\end{tabular}




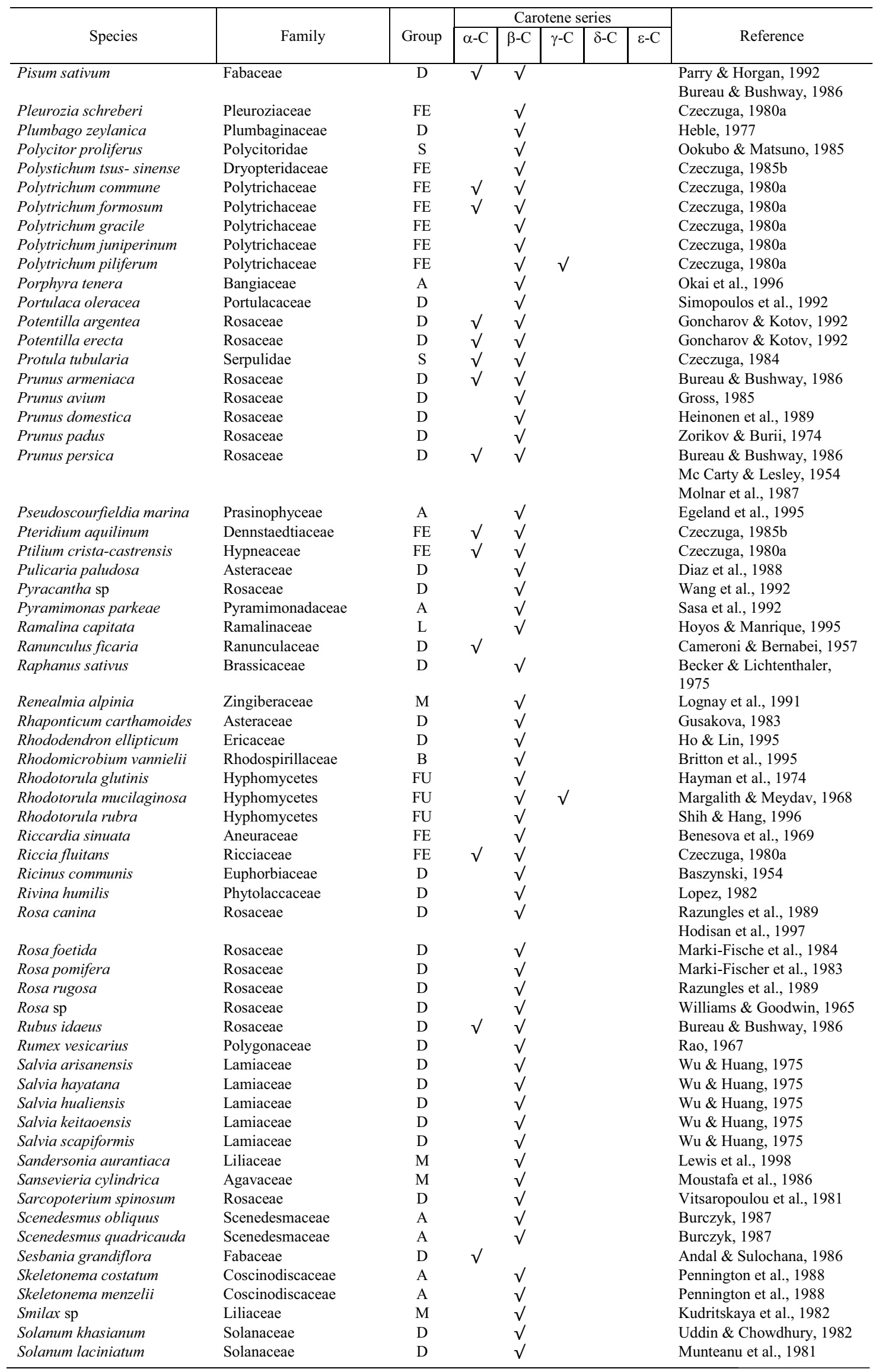




\begin{tabular}{|c|c|c|c|c|c|c|c|c|}
\hline \multirow[b]{2}{*}{ Species } & \multirow[b]{2}{*}{ Family } & \multirow[b]{2}{*}{ Group } & \multicolumn{5}{|c|}{ Carotene series } & \multirow[b]{2}{*}{ Reference } \\
\hline & & & $\alpha-C$ & $\beta-\mathrm{C}$ & $\gamma-\mathrm{C}$ & $\delta-\mathrm{C}$ & $\varepsilon-\mathrm{C}$ & \\
\hline & & & & & & & & Munteanu et al., 1980 \\
\hline Solanum nigrum & Solanaceae & $\mathrm{D}$ & $\sqrt{ }$ & & & & & Dan et al., 1982 \\
\hline Solanum persicum & Solanaceae & $\mathrm{D}$ & $\sqrt{ }$ & $\sqrt{ }$ & & & & Novruzov, 1983 \\
\hline Solanum tuberosum & Solanaceae & $\mathrm{D}$ & $\sqrt{ }$ & $\sqrt{ }$ & & & & Granado et al., 1992 \\
\hline Solorina crocea & Peltigeraceae & $\mathrm{L}$ & & $\sqrt{ }$ & & & & Czeczuga \& Olech, 1990 \\
\hline Sorbus aucuparia & Rosaceae & $\mathrm{D}$ & $\sqrt{ }$ & $\sqrt{ }$ & & & & Valadon et al., 1972 \\
\hline Sphaerophorus globosus & Sphaerophoraceae & $\mathrm{L}$ & & $\sqrt{ }$ & & & & Czeczuga \& Olech, 1990 \\
\hline Sphagnum apiculatum & Sphagnaceae & FE & $\sqrt{ }$ & $\sqrt{ }$ & & & & Czeczuga, 1980a \\
\hline Sphagnum magellanicum & Sphagnaceae & $\mathrm{FE}$ & $\sqrt{ }$ & $\sqrt{ }$ & & & & Czeczuga, 1980 \\
\hline Sphagnum palustre & Sphagnaceae & FE & $\sqrt{ }$ & $\sqrt{ }$ & $\sqrt{ }$ & & & Czeczuga, 1985b \\
\hline Sphagnum recurvum & Sphagnaceae & $\mathrm{FE}$ & $\sqrt{ }$ & $\sqrt{ }$ & $\sqrt{ }$ & & & Czeczuga, 1985b \\
\hline Sphagnum squarrosum & Sphagnaceae & $\mathrm{FE}$ & $\sqrt{ }$ & $\sqrt{ }$ & $\sqrt{ }$ & & & Czeczuga, 1985b \\
\hline \multirow[t]{3}{*}{ Spinacia oleracea } & Chenopodiaceae & $\mathrm{D}$ & $\sqrt{ }$ & $\sqrt{ }$ & & & & Granado et al., 1992 \\
\hline & & & & & & & & Rao, 1967 \\
\hline & & & & & & & & Bureau \& Bushway, 1986 \\
\hline Spirulina dunaliella & Oscillatoriaceae & $\mathrm{A}$ & & $\sqrt{ }$ & & & & Jenkins et al., 1993 \\
\hline Spirulina $\mathrm{sp}$ & Oscillatoriaceae & A & & $\sqrt{ }$ & & & & Schwartz et al., 1988 \\
\hline Sporobolomyces chosaticus & Hyphomycetes & $\mathrm{FU}$ & & $\sqrt{ }$ & & & & $\begin{array}{l}\text { Golyakov \& Tikhonova, } \\
1975\end{array}$ \\
\hline Sporobolomyces paraseus & Hyphomycetes & FU & & $\sqrt{ }$ & & & & $\begin{array}{l}\text { Golyakov \& Tikhonova, } \\
1975\end{array}$ \\
\hline Sporobolomyces roseus & Hyphomycetes & FU & & $\sqrt{ }$ & & & & $\begin{array}{l}\text { Golyakov \& Tikhonova, } \\
1975\end{array}$ \\
\hline Staphylococcus aureus & Micrococcaceae & $\mathrm{B}$ & & & & $\sqrt{ }$ & $\sqrt{ }$ & $\begin{array}{l}\text { Mel'nikova \& Ignatov, } 1973 \\
\text { Czecuga \& Heczko, } 1988\end{array}$ \\
\hline Stemmadenia glabra & Apocynaceae & $\mathrm{D}$ & & $\sqrt{ }$ & & & & Ciccio et al., 1982 \\
\hline \multirow[t]{3}{*}{ Stereocaulon alpinum } & Stereocaulaceae & $\mathrm{L}$ & $\sqrt{ }$ & $\sqrt{ }$ & & & & Czeczuga \& Olech, 1990 \\
\hline & & & & & & & & Czeczuga \& Alstrup, 1987 \\
\hline & & & & & & & & Czeczuga, 1986 \\
\hline Stereocaulon paschale & Stereocaulaceae & $\mathrm{L}$ & $\sqrt{ }$ & & & & & Czeczuga \& Alstrup, 1987 \\
\hline \multirow[t]{2}{*}{ Stereocaulon vesuvianum } & Stereocaulaceae & $\mathrm{L}$ & $\sqrt{ }$ & $\sqrt{ }$ & & & & Czeczuga \& Olech, 1990 \\
\hline & & & & & & & & Czeczuga et al., 1989 \\
\hline Stereocaulon vulcani & Stereocaulaceae & $\mathrm{L}$ & & $\sqrt{ }$ & & & & Czeczuga et al., 1988 \\
\hline Stevia rebaudiana & Asteraceae & $\mathrm{D}$ & & $\sqrt{ }$ & & & & Cheng \& Chang, 1983 \\
\hline Stichopus regalis & Stichopodidae & $\mathrm{S}$ & & $\sqrt{ }$ & & & & Czeczuga, 1984 \\
\hline Sticta canariensis & Lobariaceae & $\mathrm{L}$ & & $\sqrt{ }$ & & & & Czeczuga et al., 1988 \\
\hline Styela clava & Styelidae & $\mathrm{S}$ & & $\sqrt{ }$ & & & & Ookubo \& Matsuno, 1985 \\
\hline Styela plicata & Styelidae & $\mathrm{S}$ & & $\sqrt{ }$ & & & & Ookubo \& Matsuno, 1985 \\
\hline Suberites sericeus & Suberitidae & $\mathrm{S}$ & & $\sqrt{ }$ & & & & Matsuno et al., 1984a \\
\hline Symphytum officinale & Boraginaceae & $\mathrm{D}$ & & $\sqrt{ }$ & & & & Fukumoto \& Kuwano, 1975 \\
\hline Tagetes erecta & Asteraceae & $\mathrm{D}$ & & $\sqrt{ }$ & & & & Khachik et al., 1999 \\
\hline Tamus communis & Dioscoreaceae & M & & $\sqrt{ }$ & $\sqrt{ }$ & & $\sqrt{ }$ & Baraud, 1958 \\
\hline Tanacetum vulgare & Asteraceae & $\mathrm{D}$ & & $\sqrt{ }$ & & & & Banthorpem \& Justice, 1972 \\
\hline Taraxacum officinale & Asteraceae & $\mathrm{D}$ & & $\sqrt{ }$ & & & & Booth, 1964 \\
\hline Tecoma argentea & Bignoniaceae & $\mathrm{D}$ & & $\sqrt{ }$ & & & & Dixit \& Srivastava, 1992b \\
\hline Tethya aurantia & Clionidae & $\mathrm{S}$ & $\sqrt{ }$ & $\sqrt{ }$ & & & $\sqrt{ }$ & Czeczuga, 1984 \\
\hline Tetragonia tetragonoides & Aizoaceae & $\mathrm{D}$ & & $\sqrt{ }$ & & & & Lee, 1975 \\
\hline Tetraselmis $\mathrm{sp}$ & Chlamydomonadaceae & A & & $\sqrt{ }$ & & & & Egeland et al., 1995 \\
\hline Tetraselmis wettsteinii & Chlamydomonadaceae & $\mathrm{A}$ & & $\sqrt{ }$ & & & & Egeland et al., 1995 \\
\hline Thalassiosira eccentrica & Thalassiosiraceae & A & & $\sqrt{ }$ & & & & Pennington et al., 1988 \\
\hline Thalassiosira oceanica & Thalassiosiraceae & A & & $\sqrt{ }$ & & & & Pennington et al., 1988 \\
\hline Thalassiosira pseudonana & Thalassiosiraceae & A & & $\sqrt{ }$ & & & & Pennington et al., 1988 \\
\hline Thalassiosira rotula & Thalassiosiraceae & A & & $\sqrt{ }$ & & & & Pennington et al., 1988 \\
\hline Thamnolia vernicularis & Siphulaceae & $\mathrm{L}$ & & $\sqrt{ }$ & & & & Czeczuga \& Olech, 1990 \\
\hline Thelypteris palustris & Thelypteridaceae & FE & & $\sqrt{ }$ & & & & Czeczuga, 1985b \\
\hline Thespesia populnea & Malvaceae & $\mathrm{D}$ & & $\sqrt{ }$ & & & & Datta, 1968 \\
\hline Thorascophaera heimii & Coccolithophycidae & $\mathrm{S}$ & & $\sqrt{ }$ & & & & Bjornland, 1990 \\
\hline Trichosanthes cucumeroide & Cucurbitaceae & $\mathrm{D}$ & & $\sqrt{ }$ & $\sqrt{ }$ & & & $\begin{array}{l}\text { Matsuno \& Nagata, } 1971 \\
\text { Matsuno et al., } 1970\end{array}$ \\
\hline Trifolium repens & Fabaceae & $\mathrm{D}$ & $\sqrt{ }$ & & $\sqrt{ }$ & & & Sergeeva et al., 1984 \\
\hline Trigonella foenum-gracum & Fabaceae & $\mathrm{D}$ & & $\sqrt{ }$ & & & & Rao, 1967 \\
\hline Triticum durum & Poaceae & M & & $\sqrt{ }$ & & & & Lier, 1975 \\
\hline Ulex europaeus & Fabaceae & $\mathrm{D}$ & $\sqrt{ }$ & $\sqrt{ }$ & & & & Schon, 1936 \\
\hline Ulex gallii & Fabaceae & $\mathrm{D}$ & $\sqrt{ }$ & $\sqrt{ }$ & & & & Schon, 1936 \\
\hline Ulota ulophylla & Orthotrichaceae & FE & $\sqrt{ }$ & $\sqrt{ }$ & & & & Czeczuga, 1980 \\
\hline Umbilicaria arctica & Umbilicariaceae & $\mathrm{L}$ & & $\sqrt{ }$ & & & & Czeczuga \& Olech, 1990 \\
\hline Umbilicaria decussata & Umbilicariaceae & $\mathrm{L}$ & $\sqrt{ }$ & $\sqrt{ }$ & & & & Czeczuga \& Olech, 1990 \\
\hline Umbilicaria rigida & Umbilicariaceae & $\mathrm{L}$ & & $\sqrt{ }$ & & & & Czeczuga \& Alstrup, 1987 \\
\hline Umbilicaria vellea & Umbilicariaceae & $\mathrm{L}$ & $\sqrt{ }$ & $\sqrt{ }$ & & & & Czeczuga \& Olech, 1990 \\
\hline
\end{tabular}




\begin{tabular}{|c|c|c|c|c|c|c|c|c|}
\hline \multirow[b]{2}{*}{ Species } & \multirow[b]{2}{*}{ Family } & \multirow[b]{2}{*}{ Group } & \multicolumn{5}{|c|}{ Carotene series } & \multirow[b]{2}{*}{ Reference } \\
\hline & & & $\alpha-\mathrm{C}$ & $\beta-\mathrm{C}$ & $\gamma-\mathrm{C}$ & $\delta-\mathrm{C}$ & $\varepsilon-\mathrm{C}$ & \\
\hline Undaria pinnatifida & Alariaceae & A & & $\sqrt{ }$ & & & & Okuda \& Ukegawa, 1977 \\
\hline Urtica dioica & Urticaceae & $\mathrm{D}$ & & $\sqrt{ }$ & & & & Kudritsata et al., 1987 \\
\hline Usnea glabrata & Parmeliaceae & $\mathrm{L}$ & $\sqrt{ }$ & $\sqrt{ }$ & & & & Czeczuga et al., 1989 \\
\hline Usnea sulphurea & Parmeliaceae & $\mathrm{L}$ & $\sqrt{ }$ & & & & & Czeczuga \& Olech, 1990 \\
\hline Vaccinium corymbosum & Ericaceae & $\mathrm{D}$ & $\sqrt{ }$ & $\sqrt{ }$ & & & & Bureau \& Bushway, 1986 \\
\hline Vaccinium macrocarpon & Ericaceae & $\mathrm{D}$ & $\sqrt{ }$ & $\sqrt{ }$ & & & & Heinonen et al., 1989 \\
\hline Vacuolaria virescens & Vacuolariaceae & A & & $\sqrt{ }$ & & & & Fiksdahl et al., 1984a \\
\hline Verbena officinalis & Verbenaceae & $\mathrm{D}$ & & $\sqrt{ }$ & & & & Winde et al., 1961 \\
\hline Vernonia baccharoides & Asteraceae & $\mathrm{D}$ & & $\sqrt{ }$ & & & & Ugaz \& Fort, 1984 \\
\hline Verticillium agaricinum & Hyphomycetes & FU & & $\sqrt{ }$ & & & & Valadon \& Mummery, 1974 \\
\hline Viburnum opulus & Caprifoliaceae & $\mathrm{D}$ & & $\sqrt{ }$ & & & & Shapiro et al., 1992 \\
\hline Viscum album & Loranthaceae & $\mathrm{D}$ & & $\sqrt{ }$ & & & & $\begin{array}{l}\text { Neamtu \& Bodea, } 1970 \\
\text { Cornelia \& Stirban, } 1976\end{array}$ \\
\hline Vitis vinifera & Vitaceae & $\mathrm{D}$ & $\sqrt{ }$ & $\sqrt{ }$ & $\sqrt{ }$ & & & $\begin{array}{l}\text { Heinonen et al., } 1989 \\
\text { Zherebin \& Kolesnik, } 1984\end{array}$ \\
\hline Zea mays & Poaceae & M & & $\sqrt{ }$ & & & & Rao, 1967 \\
\hline
\end{tabular}

Group: $\mathrm{A}=$ Algae, $\mathrm{B}=$ Bacteria and allies, $\mathrm{D}=$ Dicotyledons, $\mathrm{FU}=$ Fungi, $\mathrm{L}=$ Lichens, $\mathrm{FE}=$ Ferns and allies, $\mathrm{FR}=$ Frogs, $\mathrm{G}=$ Gymnosperms, $\mathrm{I}=$ Insects, $\mathrm{M}=$ Monocotydedons, and $\mathrm{S}=$ Animals of the sea (annelids, crabs, echinodermates, fishes, mollusks, prawns, shellfishes, sponges and starfishes).

Carotene series: $\alpha-C=\alpha$-carotene, $\beta-C=\beta$-carotene, $\gamma-C=\gamma$-carotene, $\delta$-C $=\delta$-carotene, and $\varepsilon$-C $=\varepsilon$-carotene.

\section{REFERENCES}

Aalbersberg WGL, Hussein S, Sotheeswaran S, Parkinson S 1993. Carotenoids in leaves of Morinda citrifolia. $J$ Herbs Spices Med Plants 2: 51-55.

Aihara MS, Yamamoto HY 1968. Occurrence of antheraxanthin in two rhodophyceae Acanthophora spicifera and Gracilaria lichenoides. Phytochemistry 7: 497-499.

Almeida RN, Navarro DS, Barbosa-Filho JM 2001. Plants with central analgesic activity. Phytomedicine 8: 310-322.

Amaral FMM, Ribeiro MNS, Barbosa-Filho JM, Reis AS, Nascimento FRF, Macedo RO 2006. Plants and chemical constituents with giardicidal activity. Rev Bras Farmacogn 16(Supl.): 696-720.

Andal KR, Sulochana N 1986. Chemical examination of the seeds of Sesbania grandiflora. Fitoterapia 57: 293-294.

Angelucci E, Arima HK, Kumagai EA 1980. Annatto. 1. Preliminary data of the chemical composition.Colet Inst Technol Aliment 11: 89-96.

Aprikyan SV 1979. Angelica tatianae Bordz as a valuable feed-silage and vegetable plant. Izv S-Kh Nauk 22: 24-32.

Argoud S 1958. Oil palm fruit carotenoids. Oleagineux 13: 249-258.

Asakawa Y, Takemoto T 1978. The pungent diterpenoid of Pellia endiviaefolia. Phytochemistry 17: 153-154.

Azuine MA, Amonkar AJ, Bhide SV 1991. Chemopreventive efficacy of betel leaf extract and its constituents on 7,12-dimethylbez(a)anthracene induced carcinogensis and their effect on drug detoxification system in mouse skin. Indian J Exp Biol 29: 346-351.

Baccarini A, Bertossi F, Bagni N 1965. Carotenoid pigments in the stem of Cuscuta australis. Phytochemistry 4: 349-351.

Banaszkiewicz W, Kowalska M, Mrozikiewicz A 1963. Determination of the estrogenic activity of extracts from Calendula officinalis flowers. Ann Pharm (Poznan) 1: 53.

Banthorpe DV, Justice AW 1972. Terpene biosynthesis. Part VI. Monoterpenes and carotenoids from tissue cultures of Tanacetum vulgare. J Chem Soc Perkin Trans I: 1769.

Baraud J 1958. Carotenoids of the flowers of Calendula and of the fruits of Tamus, Rosa, Malpighia, Physalis. Rev Gen Bot 6: 221.

Barbosa-Filho JM, Vasconcelos THC, Alencar AA, Batista LM, Oliveira RAG, Guedes DN, Falcão HS, Moura MD, Diniz MFFM, Modesto-Filho J 2005. Plants and their active constituents from South, Central, and North America with hypoglycemic activity. Rev Bras Farmacogn 15: 392-413.

Barbosa-Filho JM, Piuvezam MR, Moura MD, Silva MS, Lima KVB, Cunha EVL, Fechine IM, Takemura OS 2006a. Anti-inflammatory activity of alkaloids: A twenty-century review. Rev Bras Farmacogn 16: 109-139.

Barbosa-Filho JM, Medeiros KCP, Diniz MFFM, Batista LM, Athayde-Filho PF, Silva MS, Cunha EVL, Almeida JRGS, Quintans-Júnior LJ 2006b. Natural products inhibitors of the enzyme acetylcholinesterase. Rev Bras Farmacogn 16: 258-285

Barbosa-Filho JM, Martins VKM, Rabelo LA, Moura MD, Silva MS, Cunha EVL, Souza MFV, Almeida RN, Medeiros IA 2006c. Natural products inhibitors of the angiotensin converting enzyme (ACE). A review between 1980-2000. Rev Bras Farmacogn 16: 421-446.

Barbosa-Filho JM, Nascimento-Júnior FA, Tomaz ACA, Athayde-Filho PF, Silva MS, Cunha EVL, Souza MFV, Batista LM, Diniz MFFM 2007. Natural products with antileprotic activity. Rev Bras Farmacogn 17: 141-148.

Barth MM, Zhou C, Kute KM, Rosenthal GA 1995. Determination of optimum conditions for supercritical fluid extraction of carotenoids from carrot (Daucus 
carota L.) tissue. J Agr Food Chem 43: 2876-2878.

Baruffaldi R, Penna TCV, Colombo AJ, Pitombo R 1981. Effect of $\mathrm{pH}$ on stability of peroxidase and of carotenes in carrot (Daucus carota L.). An Farm Quim Sao Paulo 21: 52-56.

Baszynski T 1954. Vegetable oils as a source of provitamin A (beta-carotene). Acta Soc Bot Pol 23: 17.

Bauer O, Demirovska V 1982. Contribution to the chemical composition of some sorts of cabbage produced in the region of Skopje.God Zb Zemjod Fak Univ Skopje 30: 55-59.

Becker K, Lichtenthaler HK 1975. Formation of individual carotenoid components in Raphanus etioplasta in continuous far-red and white light. Z Pflanzenphysiol 7: 303 .

Ben-Amotz A, Lers A, Avron M 1990. Stereoisomers of beta-carotene and phytoene in the alga Dunaliella bardawil. Plant Physiol 86: 1286-1291.

Benesova V, Herout V, Sorm F 1969. Components of liverworts. Collect Czech Chem Commun 3: 1810-1814.

Biacs PA Daood HG, Huszka TT, Biacs PK 1993. Carotenoids and carotenoid esters from new cross-cultivars of paprika. J Agr Food Chem 411: 1864-1867.

Bjoerland T, Tangen K 1979. Pigmentation and morphology of a Marine glyrodinium (Dinophyceae) with a major carotenoid different from peridinin and fucoxanthin. J Phycol 15: 457-463.

Bjoernland T 1984. Chlorophyll a and carotenoids of the red siga Erythrotrichia carnea. Biochem Syst Ecol 12: 279-283.

Bjornland T, Guillard RRL, Jensen SL 1988. Phaeocystis sp. Clone 677-3 - a tropical marine planktonic prymnesiophyte with fucoxanthin and 19'-acyloxyfucoxanthins as chemosystematic markers. Biochem Syst Ecol 16: 445-452.

Bjornland T 1990. Chromatographic separation and spectrometric characterization of native carotenoids from the marine dinoflagellate Thoracosphaera heimii. Biochem Syst Ecol 18: 307-316.

Bladt S, Wagner H, Woo WS 1990. Analysis and standardization of plant drugs by HPLC and other chromatographic techniques. Part 13. Taiga roots. TLC and HPLC analysis of Eleutherococcus and Acanthopanax extracts and their pharmacognostic products. Dtsch Apoth Ztg 130: 1499-1508.

Bobbio FO, Scamparini ARP 1980. Carbohydrates, organic acids and carotenoids of Bromelia faustuosa Lindl. Ind Aliment 19: 768-770.

Booth VH 1964. Taraxien, the carotenoid ester in dandelion flowers. Phytochemistry 3: 229-234.

Boston HL, Farmer AM, Madsen JD, Adams MS, Hurley JP 1991. Light-harvesting carotenoids in two deepwater bryophtes. Phytosynthetica 25: 61-66.

Bramley PM, Davies BH 1975. Carotene biosynthesis by cell extracts of mutants of Phycomyces blakesleeanus. Phytochemistry 14: 463-469.

Bramley PM, Davies, BH 1976. Beta-carotene biosynthesis by extrats of the c115 mutant of Phycomyces blakesleeanus. Phytochemistry 15: 1913-1916.

Brieskorn CH, Meister G 1965. Occurrence of isosakuranetin 7-rhamnosidoglucoside in the leaf of Monarda didyma. Arch Pharm (Weinheim) 298: 435-440.

Britton G, Singh RK, Goodwin TW, Ben-Aziz A 1975. The carotenoids of Rhodomicrobium vannielii
(Rhodospirillaceae) and the effect of diphenylamine on the carotenoid composition. Phytochemistry 14: 2427-2433.

Britton G 1984. Carotenoids and polyterpenoids. Nat Prod Rep 1: 67-86.

Britton G 1985. Carotenoids and polyterpenoids. Nat Prod Rep 2: 349-388.

Britton G 1986. Carotenoids and polyterpenoids. Nat Prod Rep 3: 591-620.

Britton G 1989. Carotenoids and polyterpenoids. Nat Prod Rep 6: 359-392.

Britton G 1991. Carotenoids and polyterpenoids. Nat Prod Rep 8: 223-250.

Buchecker R, Jensen SL 1975. Carotenoids in higher plants. Part VIII. The carotenoid pattern in Iris germanica. Phytochemistry 14: 851-852.

Buchecker R, Jensen SL, Borch G, Siegelman HW 1976. Carotenoids of blue-green algae. Part 9. Carotenoids of Anacystis nidulans, structures of caloxanthin and nostoxanthin Phytochemistry 15: 1015-1018.

Buishand JG, Gabelman WH 1978. Investigation on the inheritance of root color and carotenoid contenti in carrot, Daucus carota. Diss Abstr Int B 39: 2656.

Burczyk J 1987. Cell wall carotenoids in green algae which form sporopollenins. Phytochemistry 26: 121-128.

Bureau JL, Bushway RJ 1986. HPLC determination of carotenoids in fruits and vegetables in the United States. J Food Sci 51: 128-130.

Bushway AA, Serreze DV, Mc Gann DF, True RH, Work TM, Bushway RJ 1985. J Food Sci 50: 1491-1516.

Calle Alvarez J, Hernandez L, Riano I, Galindo G 1986. Isolation and identification of some compounds in the oil of the pasture grass Melinis minutiflora. Rev Colomb Cienc Quim-Farm 1: 83-85.

Cameroni R, Bernabei MT 1957. The pigments of Ranunculus ficaria. Atti Soc Nat Mat Modena 87-88: 125-131 .

Ceruti A, Fiussello N, Luppi Mosca AM 1972. Flavonoids in Lotus corniculatis petals in relation to altitude. Atti Accad Sci Torino Cl Sci Fis Mat Natur 10: 333.

Chalukova M, Zagorska N, Georgieva R, Abadzhieva M, Dimova P 1985. Carotene composition of fruits from regenerants obtained from Lycopersicon cheesmanii var. minor (Hook.) Mull. Dokl Bolg Akad Nauk 38: 239-241.

Chandra A, Nair MG 1997. Supercritical fluid carbon dioxide extraction of alpha-and beta-carotene from carrot (Daucus carota L.). Phytochem Anal 8: 244-246.

Chaplinskaya MG 1956. The composition of Hypericum perforatum Grass. Sbornik 269.

Chen J, Lin ZM, Jin S, Xing Q 1995. Flavor composition of Dunaliella salina. Beijing Daxue Xuebao Ziran Kexueban 31: 383-386.

Cheng TF, Chang WH 1983. Studies on the nonstevioside components of Stevia extracts. $\mathrm{K}^{\prime} \mathrm{O}$ Hsueh Fa Chan Yueh K'an 11: 96-108.

Chernenko TV, Umarov AU 1974. Unsaponifiable substances in the oil of Hibiscus syriacus seeds. Khim Prir Soedin 10: 652 .

Ciccio JF, Castro VH, Acuna F 1982. Compounds from the leaves and fruit of Stemmadenia glabra Benth. An Quim 7: 135-136.

Collier A, Lemaire B 1974. Carotenoids of argan oil. Cah Nutr Diet 94: 300-301.

Cornelia D, Stirban M 1976. Annual dynamics of assimilatory 
pigments in Viscum album L. and in its host plant, Populus tremula. Contrib Bot Gradina Bot Univ Babes-Bolyai Cluj 243.

Coxon DT, Curtis RF, Price KR, Levett G 1973. Abnormal metabolites produced by Daucus carota roots stored under conditions of stress. Phytochemistry 12: 1881-1885.

Cyunel E, Czygan FC 1989. Carotenoids of Basella alba L. Plant and its callus tissue. Herba Pol 35: 79-83.

Czeczuga B 1975. Carotenoids in the crab, Carcinus maenas of ofotfjord. Comp Biochem Physiol Ser B 51: 309-311.

Czeczuga B 1978. Carotenoids in fish. VIII. Gadidae from polish waters. Rocz Nauk Roln Ser H 9: 47-53.

Czeczuga B 1980a. Investigations on carotenoids in Embryophyta. I. Bryophyta. Bryologist 8: 21-28.

Czeczuga B 1980b. Investigations on carotenoids in lichens. 3. Species of Peltigera willd. Cryptogam Bryol Lichenol 1: 189-196.

Czeczuga B 1981. Carotenoids in fish. XXX. Rhodoxanthin in Ctenopharyngodon idella Val. (Cyprinidae). Comp Biochem Physiol Ser B 69: 885-887.

Czeczuga B 1982. Carotenoids in fish. XXXIII. Cyclopterus lumpus Linne (Cyclopteridae). Zool Pol 29: 33-36.

Czeczuga B, Ruprecht AL 1983. Investigations on carotenoids in amphibia. IV. The presence of antheraxanthin in specimens of Bufo calamita Laurenti. Folia Biol (Krakow) 31: 349-353.

Czeczuga B 1984. Investigations of carotenoids in some animals of the Adriatic Sea-VI. Representatives of sponges, annelids, molluscs and echinodermates. Comp Biochem Physiol Ser B 78: 259-264.

Czeczuga B 1985a. Carotenoids in representatives of the Cladoniaceae. Biochem Syst Ecol 13: 83-88.

Czeczuga B 1985b. Carotenoids in sixty-six representatives of the Pteridophyta. Biochem Syst Ecol 13: 221-230.

Czeczuga B 1986. Investigations on carotenoids in lichens. XI. Lichens from Lapland. Ann Bot Fenn 23: 251-254.

Czeczuga B 1987. Ketocarotenoids - autumn carotenoids in Metasequoia glyptostroboides. Biochem Syst Ecol 15: 303-306.

Czeczuga B, Alstrup V 1987. The carotenoid content of lichens from Greenland. Biochem Syst Ecol 15: 297-301.

Czeczuga B 1988. Investigations on carotenoids in insects. IX. Apocarotenals in representatives of the cerambycidae family. Folia Biol (Krakow) 36: 167-172 .

Czecuga B, Heczko P 1988. Carotenoids in Staphylococcus aureus cells. Rocz Acad Med Im Juliana Marchlewskiego Bialymstorku 29: 1984-1985.

Czeczuga B, Cifuentes B, Reynaud PA 1988. Carotenoids in lichens from the Canary Islands. Biochem Syst Ecol 16: 117-118.

Czeczuga B, Caccamese S, Passadore MV 1989. Investigations on carotenoids in lichens: XX. Carotenoids in lichens from various italian environments. Phyton (Austria) 29: $15-22$.

Czeczuga B, Olech M 1990. Investigations on carotenoids in lichens. XXV. Studies on carotenoids in lichens from Spitsbergen. Phyton 30: 235-245.

Czinkotai B, Daood GH, Biacs PA, Hajdu F 1989. Separation and detection of paprika pigments by HPLC. J Liq Chromatogr 121: 2707-2717.

Czygan FC, Grunsfelder M 1975. Carotenoids of the porterlincoln-series in Anthurus archeri. Z Naturforsch 30c: 297

Czygan FC, Herboth H 1976. Plant tissue culture as an objective of pharmaceutical biological research. Osterr Apoth-Ztg 30: 461.

Dan S, Dan SS, Mukhopadhayay P 1982. Chemical examination of three indigenous plants. J Indian Chem Soc 59: 419-420.

Daraseliya GA, Bochoridze LD 1979. Carotenoid pigments of Mycobacterium brevicale. Mikrobiol Zh (Kiev) 41: 685-687.

Datta SC, Murti VVS, Seshadri TR 1968. A new component of the flowers of Thespesia populnea: gossypol. Curr Sci 37: 135.

David HL 1974. Biogenesis of beta-carotene in Mycobacterium kansasii. J Bacteriol 11: 386.

Davidson BS, Eisner T, Meinwald J 1991. 3,4-Didehydrobeta,beta-caroten-2-one, a new carotenoid from the eggs of the stick insect Anisomorpha buprestoides. Tetrahedron Lett 32: 5651-5654.

Deli J, Matus Z, Toth G 1996. Carotenoid composition in the fruits of Capsicum annuum CV. Szentesi kosszarvu during ripening. J Agr Food Chem 44: 711-716.

Desai HG, Desai JD, Modi VV 1975. Involvement of biotin in carotene formation by Phycomyces blakesleeanus and Blakeslea trispora. Curr Sci 44: 619.

Diaz N, Ortega T, Pardo MP 1988. Pharmacognostic study of Pulicaria paludosa Link. An R Acad Farm 54: 526-531.

Dixit BS, Srivastava SN 1992a. Flavonoids and carotenoids of Cochlospermum vitifoleum flower. Fitoterapia 63: 270.

Dixit BS, Srivastava SN 1992b. Flavonoids and carotenoids of Tecoma argentea flowers. Fitoterapia 63: 272.

Doiiode SD, Sulledmath UV 1986. Genetic variability and correlation studies in pumpkin (Cucurbita moschata Poir.) Mysore J Agr Sci 20: 59-61.

Donkin P 1976 . Ketocarotenoid biosynthesis by Haematococcus lacustris. Phytochemistry 15: 711.

Duquenois P 1972. Safrron in the modern pharmacy. Identification and purity analyses. Bull Soc Pharm 15: 149 .

Ebert G, Gross J 1985. Carotenoid changes in the peel of ripening persimmon (Diospyros kaki) cv triumph. Phytochemistry 24: 29-32.

Egeland ES, Eikrem W, Throndsen J, Wilhelm C, Zapataii M, Jensen SL 1955. Biochem Syst Ecol 23: 747-755.

Elahi M, Glass RW, Lee TC, Chichester CO, Simpson KL 1975. The effect of cpta analogs and other nitrogenous compounds on the biosynthesis of carotenoids in Phycomyces blakesleeanus mutants. Phytochemistry 14: 133-138.

El-Fifrawi EA, Hudson BJF 1979. Identification and estimation of carotenoids in the seeds of four Lupinus species. $J$ Agr Food Chem 30: 1168-1170.

Eng-Wilmont DL, Martin DF 1979. The chemistry of marine blue-green alga, Gomphosphaeria aponina. Growth and production of the bioactive natural product aponin. Diss Abstr Int B 39: 3319-3320.

Eslava AP, Alvarez MI, Cerda-Olmedo E 1974. Regulation of carotene biosynthesis in phycomyces by vitamin A and beta-ionone. Eur J Biochem 4: 617.

Falcão HS, Lima IO, Santos VL, Dantas HF, Diniz MFFM, Barbosa-Filho JM, Batista LM 2005. Review of the plants with anti-inflammatory activity studied in 
Brazil. Rev Bras Farmacogn 15: 381-391.

Federmann S, Federmann M 2000. Estratégias nutricionais eficazes na prevenção do câncer, doenças cardiovasculares, diabetes entre outras, $2^{\text {a }}$ Ed. Iglu Editora, São Paulo.

Feofilova EP, Shakhova IK, Kudryavtseva NN 1974. Carotenogenic proteins of Blakeslea trispora studies by disc eletrophoresis in polyacrylamide gel. Mikrobiologiya 43: 235.

Feofilova EP, Red'kina TV 1975. Effect of high hydrostatic pressure on carotenoid level in the mycelium of Blakeslea trispora. Mikrobiologiya 44: 180.

Fiksdahl A, Withers N, Guillard RRL, Jensen SL 1984a. Algal carotenoids. Part 31. Carotenoids of the Raphidophyceae chemosystematic contribution. Comp Biochem Physiol Ser B 76: 265-271.

Fiksdahl A, Withers N, Jensen SL 1984b. Carotenoids of Heterosigma akashiwo: a chemosystematic contribution. Biochem Syst Ecol 12: 355-356.

Findlay JA, Patil AD 1984. Antibacterial constituents of the diatom Navicula delognei. J Nat Prod 47: 815-818.

Findlay JA, Patil AD 1985. Sterol and other constituents of the brown alga Desmarestia aculeata. Phytochemistry 24: 366-367.

Findlay JA, Patil AD 1986. Antibacterial constituents of the red alga Cystoclonium purpureum. Phytochemistry 25: 548-550.

Fishman GM, Chikovani DM 1990a. Characterization of the carotenoid complex of the leaves of different varieties of subtropical persimmon. Chem Nat Comp 26: 344.

Fishman GM, Chikovani DM 1990b. Identification of the carotenoids of the leaves of Camellia sasanqua. Chem Nat Comp 26: 227.

Foss P, Skulberg OM, Kilaas L, Jensen SL 1986. The carbohydrate moieties bound to the carotenoids myxol and oscillol and their chemosystematic applications. Phytochemistry 25: 1127-1132.

Fraczek T, Bubicz M, Grochowski M 1977. Carotene, alphatocopherol, and 1-ascorbic acid content of celery (Apium graveolens foliosum). Przem Spozyw 31: 438.

Francis GW, Hertzberg S, Andersen K, Jensen SL 1970. New carotenoid glycosides from Oscillatoria limosa. Phytochemistry 9: 629-635.

Francis GW, Strand LP, Lien T, Knutson G 1975. Variations in the carotenoid content of Chlamydomonas reinhardii throughout the cell cycle. Arch Microbiol 10: 249.

Francis GW, Isaken M 1989. Droplet counter current chromatography of the carotenoids of parsley Petroselinum crispum. Chromatographia 27: 549-551.

Fresnedo O, Gomez R, Serra J 1991. Carotenoid composition in the Cyanobacterium phormidium laminosum. Effect of nitrogen starvation. Febs Lett 282: 300-304.

Fukumoto K, Kuwano T 1975. Collecting xanthophyll, sterol, carotene and phytol from comfrey. PatentJapan-75,10,320: 3pp.

Gachechiladze ND, Korzinnikov YS, Glazunova EM, Yusufbekov KY, Bondar VV, Krymskaya NB, Apova IM 1981. Biochemical and morphological characterization of the forms of sea Buckthorn growing in the Western Pamir. Rast Resur 17: 37-42.

Gaind KN, Juneja TR 1970. Capparis decidua. Phytochemical study of flowers and fruits. Res Bull Paniab Univ Sci 211: 67-71.

Getahun A, Der Krikorian A 1973. Chat: coffee's rival from Harar, Ethiopia. I. Botany, cultivation and use. Econ Bot 27: 353-377.

Giri J, Bhuvaneswari V, Tamilarasu R 1980. Evaluation of the nutritive content of five varieties of papaya in different stages of ripening. Indian J Nutr Diet 17: 319-325.

Golubev VN, Gusar ZD, Mamedov ES 1986. Pigments of Olea europea. Chem Nat Comp 22: 225-226.

Golyakov PN, Tikhonova TN 1975. Carotenoid pigments of Sporobolomyces genus yeasts. Mikol Fitopatol 9: 193.

Gonçalves MCR, Moura LSA, Rabelo LA, Cruz HMM, Cruz J, Barbosa-Filho JM 2000. Produtos naturais inibidores da enzima HMG CoA redutase. Rev Bras Farm 81: 63-71.

Goncharov NF, Kotov AK 1992. Coumarins, carotenoids, and beta-sitosterol from the epigeal parts of some species of the Genus potentialla. Chem Nat Comp 27: 752.

Goodwin TW 1952. Carotenoids of the berries of Lonicera japonica. Biochem J 51: 458-463.

Goodwin TW 1954. Carotenogenesis. XIII. Carotenoids of the flower petals of Calendula officinalis. Biochem $J 5$ : 90.

Goodwin TW, Thomas DM 1964. The carotenoid pigments in the petals of Mimulus cupreus and Mimulus tigrinus. Phytochemistry 3: 47-50.

Granado F, Olmedilla B, Blanco I, Rojas-Hidalgo E 1992. Carotenoid composition in raw and cooked spanish vegetables. J Agr Food Chem 40: 2135-2140.

Gregory GK, Chen TS, Philip T 1987. Quantitative analysis of carotenoids and carotenoid esters in fruits by HPLC: red bell peppers. J Food Sci 52: 1071-1073.

Grill D, Pfeifhofer W, Esterbauer H 1984. Carotenoids in Chrysomyxa abietis infected spruce needles. Eur J Forest Pathol 14: 296-301.

Gross J, Gabai M, Lifshitz A 1972. The carotenoids of the avocado pear. Persea americana, nabal variety. $J$ Food Sci 37: 589-591.

Gross JA, Stroz RJ, Britton G 1975. Carotenoid hydrocarbons of Euglena gracilis and derived mutants. Plant Physiol 55: 175.

Gross J 1982. Changes of chlorophylls and carotenoids in developing strawberry fruits (Fragaria ananassa) CV. tenira. Gartenbauwissenschaft 47: 142-144.

Gross J 1985. Carotenoid pigments in the developing sweet cherry (Prunus avium) doenissen's gelbe". Gartenbauwissenschaft 50: 88-90.

Gross J, Ikan R, Eckhardt G 1983. Carotenoids of the fruit of Averrhoa carambola. Phytochemistry 22: 1479-1481.

Grung M, Jensen SL 1993. Algal carotenoids 52; secondary carotenoids of algae 3 ; carotenoids in a natural bloom of Euglena sanguinea. Biochem Syst Ecol 21: 757-763.

Gupta R, Agrawal M, Baslas RK 1980. Identification of some vitamins in the seeds of Apium graveolens. Herba Hung 19: 109-111.

Gusakova SD, Stepanenko GA, Asilbekova DT, Murdokhaev YM 1983. Lipids of some medicinal plants. Rast Resur 19: 444-455.

Hai T, Scheneider B, Schmidt J, Adam G 1996. Sterols and 
triterpenoids from the Cyanobacterium anabaena Hallensis. Phytochemistry 41: 1083-1084.

Hallegraeff GM, Nichols PD, Volkman JK, Blackbur SI, Everitt DA 1991. Pigments, fatty acids, and sterols of the toxic Dinoflagellate Gymnodinium catenatum. J Phycol 27: 591-599.

Halsall TG, Hills IR 1971. Isolation of heneicosa1,6,9,12,15,18-hexaene and -1,6,9,12,15-pentaene from the alga Fucus vesiculosus. Chem Commun 448-449.

Hanny BW, Hedin PA 1972. Phytochemical studies in the family Malvaceae. II. Analysis of some chemical constituents of four Hibiscus species.Diss Abstr Int $B$ 33: 1424a.

Hanny BW, Henson RD, Thompson AC, Gueldner RC, Hedin PA 1972. Identification of carotenoid constituents in Hibiscus syriacus. J Agr Food Chem 20: 914.

Haugan JA, Jensen SL 1994. Algal carotenoids 54. Carotenoids of brown algae (Phaeophyceae). Biochem Syst Ecol 22: 31-41.

Hayman EP, Yokoyama H, Chichester CO, Simpson KL 1974. Carotenoid biosynthesis in Rhodotorula glutinis. $J$ Bacteriol 120: 1339.

Heble MR 1977. Plant tissue cultures, a source of natural products: biosynthesis of steroids and naphthoquinones.Cultiv Util Med Aromat Plants 510-514.

Heinonen MI, Ollialainen V, Linkola EK, Varo PT, Koivistoinen PE 1989. Carotenoids in finnish foods: vegetables, fruits, and berries. J Agr Food Chem 37: 655-659.

Hertzberg S, Jensen SL 1966. The carotenoids of blue-green algae-II. The carotenoids of Aphanizomenon flosaquae. Phytochemistry 5: 565-570.

Hertzberg S, Bergquist P, Jensen SL 1989. Further occurrence of sulphated carotenoids in Ianthella species (Desmospongia). Biochem Syst Ecol 17: 51-53.

Ho LK, Lin WN 1995. Quercetin 5,4'-dimethyl ether from Rhododendron ellipticum Phytochemistry 39: 463-464.

Hodisan T, Socacaiu C, Ropan I, Neamtu G 1997. Carotenoid composition of Rosa canina fruits determined by thin-layer chromatography and high-performance liquid chromatography. J Pharm Biomed Anal 16: 521-528.

Hoque E 1988. Isocratic reversed-phase high-performance liquid chromatographic analysis of pigments in norway spruce. J Chromatogr 44: 417-423.

Hoyos MAS, Manrique E 1995. Effect of nitrate and ammonium ions on the pigment content (xanthophylls, carotenes and chlorophylls) of Ramalina capitata. Lichenologist 27: $155-160$.

Ignasiak T, Lesins K 1975. Carotenoids in petals of perennial Medicago species. Biochem Syst Ecol 2: 177.

Imamura H, Umehara K, Ohashi H 1980. Constituents of Caesalpinia japonica (Leguminosae). Gifu Daigaku Nogakubu Kenkyu Hokoku 4: 75-82.

Isler O 1971. Carotenoids. Birkhauser Verlag, Basel, pp. 932.

Jenkins MY, Sheikh NM, Mitchell GV, Grundel E, Blakely SR, Carter CJ 1993. Dietary carotenoids influenced biochemical but not morphological changes in adult male rats fed a choline-deficient diet. Nutr Cancer 19: $55-65$.

Jiang WD 1989. Comparative studies on quality between the wild and cultivated Hedysarum polybotrys roots from sichuan province. Chung Ts'ao Yao 20: 373-374.

Jones LA 1979. Gossypol and some other terpenoids, flavonoids, and phenols that affect quality of cottonseed protein. J Amer Oil Chem Soc 56: 727-730.

Joyce AE 1954. Some polyenes from Brassica rutabaga. Nature 17: 311-312.

Jui D, Feibelman T, Bennett JW 1998. A preliminary study of the carotenoids of some North American species of Cantharellus. Int J Plant Sci 15: 244-248.

Karag'ozova MD 1974. Histological and histochemical studies of several medicinal plants. Farmatsiya (Sofia) 24: 26.

Kasemsri K, Intararaksa B, Wikiniyathanee W 1952. Quantitative analysis of carotene in Ipomora aquatica Forsk.Undergraduate Special Project Report 16pp.

Katagiri K, Koshino Y, Maoka T, Matsuno T 1987. Investigations on carotenoids in lichens. 3. Species of Peltigera willd. Comp Biochem Physiol Ser B 87: 161-163.

Kayser H 1975. The use of argentation chromatography for the analysis of fatty acid esters of polyenes: the structure of carotenoid esters of Aglais urticae (Lepidoptera, Insecta). Z Naturforsch 30c: 369.

Khachik F, Steck A, Pfander H 1999. Isolation and structural elucidation of $\left(13 \mathrm{Z}, 13^{\circ} \mathrm{Z}, 3 \mathrm{R}, 3^{`} \mathrm{R}, 6^{`} \mathrm{R}\right)$-lutein from marigold flowers, kale, and human plasma. $J$ Agr Food Chem 47: 455-461.

Khodzhaev AS 1981. Carotenoids of cotton leaves and their quantitative changes during the ontogenetic development of cotton. Carotenoids of cotton leaves and their quantitative changes during the ontogenetic development of cotton. Uzb Biol Zh 5: 20-23.

Kitayama M, Takakura I 1991. Manufacture of beta-carotene with freshwater green algae. Patent Japan Kokai Tokkyo Koho-03 183,498: 5pp

Koch L, Madl F, Schlick P, Matits S, Toth K, Horvath, Pinter L, Fodor L 1991. Beta-carotene concentrate from carrot. Patent-Hung Teljes-55,752 :12pp.

Kudritsata SE, Filman GM, Zagorodskaya LM, Chikovanii DM 1987. Carotenoids of Urtica dioica. Chem Nat Comp 22: 604-605.

Kudritskaya SE, Fishman GM, Zagorodskaya LM, Chikovani DM 1983. Carotenoids of promising Citrus hybrids. Khim Prir Soedin 19: 111-112.

Kudritskaya SE, Fishman GM, Chikovani DM 1984. Carotenoids of the fruit of the subtropical persimmon, variety khachia. Chem Nat Comp 20: 369.

Kudritskaya SE, Fishman GM, Zagorodskaya LM, Chikovani DM 1988a. Carotenoids from leaves of Mimosa biuncifera. Chem Nat Comp 24: 258.

Kudritskaya SE, Fishman GM, Zagorodskaya LM, Chikovani DM 1988b. Carotenoids of Orthosiphon stamineus. Chem Nat Comp 23: 767-768.

Kuhn R, Winterstein A 1933. Picrocrocin the terpene glucoside of safran and the biogenesis of the carotenoid carboxylic acids. Naturwissenschaften 2: 527.

Kushwaha SC, Kramer JKG, Kates M 1975. Isolation and characterization of C-50-carotenoid pigments and other polar isoprenoids from Halobacterium cutirubrum. Biochim Biophys Acta 39: 303.

Kutateladze DS 1974. Seasonal dynamics of the levels of plastid pigments in leaves of various mandarin varieties and hybrids. Subtrop Kul't 2: 51.

Laddha KS, Jolly CI 1985. Preliminary phytochemical studies 
on the leaves of Capparis zeylanica Linn. Indian Drugs 22: 499.

Lagazidze DS, Murav'eva DA, Bostoganashvili VS 1984. Content of pharmacologically active compounds in oil from fruit pulp of Hippophae rhamnoides grown in Georgia[USSR]. Khim Farm Zh 18: 713-717.

Laval-Martin C, Quennemet J, Moneger R 1975. Pigment evolution in Lycopersicon esculentum fruits during growth and ripening. Phytochemistry 14: 2357-2362.

Lee C 1975. Studies on the chemical composition of Tetragonia tetragonides. Proc Nat Sci Counc Part 1 (Taiwan) 8: 145.

Lee TC, Rodriguez DB, Karasawa I, Lee TH, Simpson KL, Chichester CO 1975. Chemical alteration of carotene biosynthesis in Phycomyces blakesleeanus and mutants. Appl Microbiol 30: 988.

Lewis DH, Bloor SJ, Schwinn KE 1998. Flavonoid and carotenoid pigments in flower tissue of Sandersonia aurantiaca (Hook.). Sci Hort 72: 179-192.

Li XH, He SN, Ren BR, Shen N 1997. Nutrient constituents of Orychophragmus violaceus (L.) O.E.Schulz and the evaluation as a wild vegetable. Zhiwu Ziyuan Yu Huanjing 6: 8-12.

Liaaen S, Sorensen NA 1955. Postmortem changes in the carotenoids of Fucus vesiculosus. Intern Seaweed Symposium 2nd Trondheim, 25.

Lichtenthaler HK, Straub V, Grumbach KH 1975. Unequal formation ofprenyl-lipids in a plant tissue culture and in leaves of Nicotiana tabacum. Plant Sci Lett 4: 61.

Lier JB 1975. Carotenoids of durum wheat. Diss Abstr Int B 35: 4369.

Lin DH, Li CX, Zhang H, Li H 1979. Effect of industrial wastewater on photosynthetic pigments in Hydrilla verticillata L. Yun-Nan Chih Wu Yen Chiu 12: 62-65.

Lognay G, Marlier M, Severin M, Haugruge E, Gibon V, Trevejo E 1991. On the characterization of some terpenes from Renealmia slpinia Rott. (Mass) oleoresin. Flavour Frag J 6: 87-91.

Lopez LFE 1982. Natural pigments: phytochemistry of Rivinia humilis. First Latinamerican \& Caribbean Symposium on Pharmacologically Active Natural Products, Havana, Cuba June 21-28 1980 Unesco, 169.

Lopez-Hernandez J, Vazquez-Oderiz L, Vazquez-Blanco E, Romero-Rodriguez A, Simal-Lozano J 1993. HPLC determination of major pigments in the bean Phaseolus vulgaris. J Agr Food Chem 41: 1613-1615.

Malachi T, Gross J, Lifshitz A, Sklarz B 1974. Flavedo carotenoid pigments of the ripe Washington-navel orange. Lebensm Wiss Technol 7: 330.

Mangayarkarasi A, Nagarajan S 1984. Chemical examination of the leaves of Millingtonia hortensis Linn. Indian Drugs 21: 420-422.

Manorama R, Rukmini C 1992. Crude palm oil as a source of beta-carotene. Nutr Res Suppl 12: S223-S232.

Maoka T, Tsushima M, Matsuno T 1989. New acetylenic carotenoids from the starfishes Asterina pectinifera and Asterias amurensis. Comp Biochem Physiol Ser B 93: 829-834.

Margalith P, Meydav S 1968. Some observations on the carotenogenesis in the yeast Rhodotorula mucilaginosa. Phytochemistry 7: 765-768.

Mariath JGR, Lima MCC, Santos LMP 1989. Vitamin A activity of buriti (Mauritia vinifera Mart) and its effectiveness in the treatment and prevention of xerophthalmia. Amer J Clin Nutr 49: 849-853.

Marius C, Neamt G, Stanescu U 1994. Constituents of Helianthemum nummularium. Fitoterapia 65: 181.

Marki-Fischer E, Marti U, Buchecker R, Eugster CH 1983. Carotenoids from hips of Rosa pomifera: discovery of (5Z)-neurosporene: synthesis of $(3 R, 15 Z)$ rubixanthin. Helv Chim Acta 66: 494-513.

Marki-Fischer E, Buchecker R, Eugster CH 1984. Reinvestigation of the carotenoids from Rosa foetida, structures of 12 novel carotenoids, stereoisomeric luteoxanthis, auroxanthins, latoxanthins and latochromes. Helv Chim Acta 67: 2143-2154.

Martin FW, Ruberte R 1975. Carotenoid pigments of Dioscorea cayenensis. Ann Appl Biol 80: 317.

Matile P, Flach BMP, Eller BM 1992. Autumn leaves of Ginkgo biloba L.: optical properties, pigments and optical brighteners. Bot Acta 105: 13-17.

Matsuno T, Ito T, Taniguchi T, Takeda S, Hirota S 1970. Sterols from fruits of Trichosanthes cucumeroides and T. japonica. Yakugaku Zasshi 90: 248.

Matsuno T, Nagata S 1971. Sterols from fruits of Trichosanthes cucumeroides and T. japonica. Phytochemistry 10: 1949.

Matsuno T, Sakaguchi S 1984. Isolation of peridinin from the sea squirt Botrylloides violaceus, "itaboya" in japanese. Nippon Suisan Gakkaishi 50: 1267.

Matsuno T, Maoka T, Katagiri K, Komori T 1984a. A new carotenoid, isorenieradicistene from the sea sponge, Suberites sericeus. Nippon Suisan Gakkaishi 50: 1071-1075.

Matsuno T, Ookubo M, Nishizawa T, Shimizu I 1984b. Carotenoids of sea squirts. I. New marine carotenoids, halocynthiaxanthin and mytiloxanthinone from Halocynthia roretzi. Chem Pharm Bull 32: 4309-4315.

Matsuno T, Maoka T 1988. Carotenoids of crustacea-VI. The carotenoids of crab Paralithodes brevipes (Hanasakigani in Japanese). Nippon Suisan Gakkaishi 54: 1437-1442.

Matsuno T, Tsushima M 1989. Carotenoids of shellfishes-X. Reductive metabolic pathways of echinenone and fritschiellaxanthin in the spindle shell Fusinus perplexus. Comp Biochem Physiol Ser B 92: 189-193.

Matsuno T, Watanabe T, Maoka T, Takemura Y 1990 Carotenoids of crustacea. VII. Carotenoids in the sea louse Ligia exotica (Crustacea: Isopoda). Comp Biochem Physiol Ser B 95: 759-761.

Mc Carty CD, Lesley JW 1954: The carotenoids, amygdalin content, and titratable acidity of white-yellow-fleshed peaches within a nearly isogenic line. Proc Amer Soc Hort Sci 64: 289-292.

Mc Cormick JP 1982. Secondary metabolites of Gossypium: a biogenetic analysis. Acs Symp Ser 189: 275-300.

Meimban EJ, Balagot AH, Parawan LC, Bautista III JG 1983. Carotenoids of Philippine mango (Mangifera indica L.), carabao variety. Philippine J Food Sci Technol 7: 3-9.

Mel'nikova GY, Ignatov VV 1973. Regulation of carotenoid biosynthesis in Staphylococcus aureus. Probl Regul 
Obmena Veshchestv Mikroorg (Bobyk, Ma Et Al Eds). Akad Nauk SSSR Nauchn Tsentr Biol Issled Puschino-On-Oka USSR 338.

Menghini A, Capuccella M, Pagiotti R, Pocceschi N, Spigarelli M 1992. Pigment content and methyl chavicol production in Agastache foeniculum Kuntze cultured in vitro. J Essent Oil Res 45: 483-486.

Mercadante AZ, Britton G, Rodriguez-Amaya DB 1998a. Carotenoids from yellow passion fruit (Passiflora edulis). J Agr Food Chem 46: 4102-4106.

Mercadante AZ, Ridriguez-Amaya DB 1998b. Effects of ripening, cultivar differences, and processing on the carotenoid composition of mango. J Agr Food Chem 48: 128-130.

Mok MC, Gabelman WH, Skoog F 1976. Carotenoid synthesis in tissue cultures of Daucus carota. J Amer Soc Hort Sci 10: 442 .

Mokady S, Abramovici A, Cogan U 1989. The safety evaluation of Dunaliella bardawil as a potential food supplement. Food Chem Toxicol 27: 221-226.

Molnar P, Szabolcs J, Radics L 1987. The constitution and absolute stereochemistry of persicaxanthin. Phytochemistry 26: 1493-1496.

Moon HI, Kwon HC, Zee OP, Lee KR 1999. Phytochemical constituents of Artemisia sylvatica Max. Korean $J$ Pharmacogn 30: 87-91.

Morais LCSL, Barbosa-Filho JM, Almeida RN 2003. Plants and bioactives compounds for the treatment of Parkinson's desease. Arquivos Brasileiros de Fitomedicina Cientifica 1: 127-132.

Morgan RC 1966. Chemical studies on concentrated pineapple juice. I. Carotenoid composition of fresh pineapples. J Food Sci 31: 213

Mosquera MIM, Jaren-Galan MJ, Garrido-Fernandez J 1992. Color quality in paprika. J Agr Food Chem 40: 2384-2388.

Mosquera MIM, Hornero-Mendez D 1993. Separation and quantification of the carotenoid pigments in red peppers (Capsicum annuum L.), paprika, and oleoresin by reversed-phase HPLC. J Agr Food Chem 41: 1616-1620.

Mosquera MIM, Jan-Galan M, Garrido-Fernandez J 1994. Influence of the industrial drying processes of pepper fruits (Capsicum annuum CV. bola) for paprika on the carotenoid content. J Agr Food Chem 42: 1190-1193.

Moura MD, Torres AR, Oliveira RAG, Diniz MFFM, BarbosaFilho JM 2001. Natural products inhibitors of models of mammary neoplasia. Brit $J$ Phytotherapy 5: 124-145.

Moura MD, Silva JS, Oliveira RAG, Diniz MFFM, BarbosaFilho JM 2002. Natural products reported as potential inhibitors of uterine cervical neoplasia. Acta Farm Bonaerense 21: 67-74.

Moustafa SMI, Kadry HA, El-Olemy MM, Bisher MM 1986. Lipids, pigments and saponins of Sansevieria cylindrica Bojer. Bull Pharm Sci Assiut Univ 9: $1-10$.

Movchan SD 1960. Carotene pigments in petals of Calendula officinalis. Zh Prikl Khim (Leningrad) 3: 484.

Munteanu E, Osianu D, Bodea C 1980. Studies on carotenoid pigments synthesized in vitro by the callus tissue of Solanum laciniatum Ait. Bul Inst Agron Cluj-Napoca Ser Agric 34: 65-67.
Munteanu E, Osianu D, Bodea C 1981. Studies on carotenoid pigments in Solanum laciniatum Ait. and tissue cultures of this plant. Stud Cercet Biochim 24: 81-84.

Neamtu G, Bodea C 1970. Chemotaxonomic studies on higher plants. III. Carotenoids of Viscum album. Stud Cercet Biochim 13: 59.

Neamtu G, Stanescu U, Tabacaru C 1990. Phytochemical researches on higher plants. III. Carotenoid and chlorophyll content in Curcubia species and varieties. Stud Cercet Biochim 33: 111-116.

Nishi A, Yoshida A, Mori M, Sugano N 1974. Isolation of variant carrot cell lines with altered pigmentation. Phytochemistry 13: 1653-1656.

Njoku OU, Eneh FU, Nwanguma BC 1997. Investigation of test lipids of Gnetum africanum. J Pharm Res Dev 22: 8-10.

Novruzov EN 1983. Chemical composition of ripe fruit from Solanum persicum Willd. Ex Roem et Schult. from Azerbaijan. Rast Resur 19: 89-92.

O'Connor WF, Drumm PJ 1941. Pigments of Iris pseudacorus (water flag). Nature 147: 58-59.

Okai Y, Higashi-Okai K, Yano Y, Otani S 1996. Identification of antimutagenic substances in an extract of edible red alga, Porphyra tenera (asadusa-nori). Cancer Lett 100: 235-240.

Okuda H, Ukegawa K 1977. The study of Undaria pinnatifida. Part I. In the case of the general tissue of Undaria pinnatifida and $\beta$-carotene. Hiroshima Jogakuin Daigaku Ronshu 27: 131-144.

Olson JA 1989. Biological actions of carotenoids. J Nutr 119: 94-95.

Omarova MA, Artamonova NA 1997. Liposoluble pigments from the herb Hypericum perforatum. Chem Nat Comp 33: 691-692.

Ookubo M, Matsuno T 1985. Carotenoids of sea squirts-II. Comparative biochemical studies of carotenoids in sea squirts. Comp Biochem Physiol 81: 137-141.

Ortaliza IC, Del Rosario IF, Minda Caedo M, Alcaraz AP 1969. The availability of carotene in some Philippine vegetables. Philippine J Sci 9: 123.

Palermo JA, Gros EG, Seldes AM 1991. Carotenoids from three red algae of the Corallinaceae. Phytochemistry 30: 2983-2986.

Parry A, Horgan R 1992. Abscisic acid biosynthesis in roots. I. The identification of potential abscisic acid precursors, and other carotenoids. Planta 18: 185-191.

Partali V, Jensen SL, Huneck S, Khaidav T 1987. Carotenoids from flowers of Lilium pumilum. Pharmazie 42: 208.

Pennington F, Guillard RRL, Jensen SL 1988. Carotenoid distribution patterns in Bacillariophyceae (diatoms). Biochem Syst Ecol 16: 589-592.

Pereira JV, Modesto-Filho J, Agra MF, Barbosa-Filho JM 2002. Plant and plant-derived compounds employed in prevention of the osteoporosis. Acta Farm Bonaerense 21: 223-234.

Peto R, Doll R, Buckley JD, Sporn MB 1981. Can dietary beta carotene materially reduce human cancer rates. Nature 290: 201-208.

Pfander H, Schurtenberger H 1982. Biosynthesis of C20carotenoids in Crocus sativus. Phytochemistry 21: 1039-1042. 
Pfeifhofer HW, Grill D 1984. Carotenoids in spruce needles. I. Qualitative studies. Phyton (Austria) 24: 283-293.

Poling SM, Hsu WJ, Yokoyama H 1975. Structure-activity relationships of chemical inducers of carotenoid biosynthesis. Phytochemistry 14: 1933-1938.

Pollard A 1936. The isolation of carotene and sterols from the unsaponifiable matter of cocksfoot. Biochem $J 30$ : 382-386.

Pospisilova J, Toul V, Dupal R 1959. Rapid modification of the method of determining provitamin A in plants. Sbornik Ceskoslov Akad Zemedel Ved 5: 583-594.

Prodan G, Mihalache M, Florescu E, Baciu E, Visarion M, Dorobantu N, Tudor T 1973. Information on aromatic herbs from the biological and usefulness viewpoint. Lucr Stint Inst Agron Bucuresti Ser B 16: 23-30.

Quijano L, Vasquez CA, Rios T 1995. Sesquiterpene lactones and a seco-caryophyllene derivative from Montanoa karwinskii. Phytochemistry 38: 1251-1255.

Ramdahl T, Kazlauskas R, Bergouist P, Jensen SL 1981. Animal carotenoids. Part 24. Carotenoids from the marine sponge Ianthella basta. Biochem Syst Ecol 9: 211-213.

Rao CN 1967. True vitamin A value of some vegetables. $J$ Nutr Diet 4: 10.

Raszeja W, Muszynska M 1980. Phytochemical studies of Heliopsis scabra Dun. Ann Acad Med Gedanensis 10: 317-325.

Razungles A, Oszmianski J, Sapis JC 1989. Determination of carotenoids in fruits of Rosa sp. (Rosa canina and Rosa rugosa) and of chokeberry (Aronia melanocarpa). J Food Sci 54: 774-775.

Rhodes BB, Hall CV 1975. Effects of cpta 2-(4-chlorophenylthio)-triethylamine hydrochloride, temperature, and genotype on carotene synthesis in carrot leaves. Hortscience 10: 22.

Rocha LG, Almeida JRGS, Macedo RO, Barbosa-Filho JM 2005. A review of natural products with antileishmanial activity. Phytomedicine 12: 514-535.

Rodrigues P, Morais H, Mota T, Olivera S, Forgacs E, Cserhati $\mathrm{T}$ 1988. Use of HPLC and multivariate methods for the evaluation of the stability of colour pigments of paprika (Capsicum annuum) powder. Anal Chim Acta 372: 411-416.

Rodriguez DB, Raymundo LC, Lee TC, Simpson KL, Chichester CO 1976. Carotenoid pigment changes in ripening Monordica charantia fruits. Ann Bot (London) 40: 615.

Rojas NHH, Diaz RC 1977. Characterization of cantaloupe (Cucumis melo L.) carotenes. Agron Trop (Maracay Venez.) 27: 465-472.

Ronneberg H, Foss P, Ramdahl T, Borch G, Skulberg OM, Jensen SL 1980. Carotenoids of blue-green algae. Occurrence and chirality of oscillaxanthin. Phytochemistry 19: 2167-2170.

Rouseff RL, Sadler GD, Putnam TJ, David JE 1992. Determination of beta-carotene and other hydrocarbon carotenoids in red grapefruit cultivars. J Agr Food Chem 40: 47-51.

Rowe TD, Parks LM 1941. A phytochemical study of Aloe vera leaf. J Amer Pharm Ass Sci Ed 30: 262-266.

Santos MC, Bobbio PA, Rodriguez-Amaya DB 1988. Carotenoid composition and vitamin A value of rami (Bohemeria nivea) leaves. Acta Aliment 17: 33-35.
Sarma S, Sarma R 1978. Effect of gibberellic acid and coumarin on the carotenoid content of carrot (Daucus carota) root. Curr Sci 47: 163.

Sasa T, Takaichi S, Hatakeyama N, Watanabe MM 1992. A novel carotenoid ester, loroxanthin dodecenoate, from Pyramimonas parkeae (Prasinophyceae) and a chlorarachniophycean alga. Plant Cell Physiol 33: 921-925.

Sato M 1992. Solubility and extraction of beta-carotene from carrot. Kenkyu Kiyo Kagoshima Diagaku Kyoikugakubu Shizen Kagakuhen 44: 103-108.

Schon K 1936. Carotenoids. III. An isomeride of lutein isolated from the furze (Ulex europaeus). Biochem J 30: 1960-1965.

Schon K, Mesqita B 1936. Carotenoids. IV. The carotenoids of Genista tridentata. Biochem J 30: 1966-1969.

Schwartz J, Shklar G, Reid S, Trickler D 1988. Prevention of experimental oral cancer by extracts of spirulinaDunaliella algae. Nutr Cancer 11: 127-134.

Sergeeva NV, Shapiro DK, Bandyukova VA, Anikhimovskaya LV, Rizhnaya TI 1979. Carotenoids of the fruit of some varieties of Hippophae rhamnoides. Chem Nat Comp 15: 87.

Sergeeva NV, Bandyukova VA, Shapiro DK 1984. Carotenoids of pollen pellets from certain plants. Khim Prir Soedin 20: 665.

Shapiro DK, Anikhimovskaya LV, Narizhnaya TI 1981. Biochemical composition of the edible fruits of the species Lonicera L. introduced in byelorussia. Rast Resur 17: 565-568.

Shapiro DK, Anikhimovskaya LV, Narizhnaya TI, Vereskovskii VV 1983. Chemical characterization of fruit from several species of Berberis L. introduced into the BSSR. Rast Resur 19: 84-89.

Shapiro DK, Kudinov MA, Biryukova LG, Narizhnaya TL, Anikhimovskaya LV 1985. Evaluation of the chemical composition of some Galium species growing in the BSSR as potential (raw material sources). Vestsi Akad Navuk Bssr Ser Biyal Navuk 4: 24-28.

Shapiro DK, Kisilevsky IR, Moroz PA, Potopalsky AL, Kuznetsova ZP, Vasilevskaya TI, Dovnar TV, Prilishch NP, Vereskovsky VV 1992. Biochemical composition of fruit of Viburnum opulus L.growing in polessie and forest-steppe in the Ukraine. Rast Resur 28: 54-63.

Shih CT, Hang YD 1996. Production of carotenoids by Rhodotorula rubra from sauerkraut brine. Food Sci Technol 29: 570-572.

Shim KH, Sung NK, Kang KS, Choi JS, Jang CW 1994. Isolation and physicochemical properties of carotenoid pigments from orange peels. Han'guk Yongyang Siklyong Hakhoe Chi 23: 143-149.

Silva JS, Moura MD, Oliveira RAG, Diniz MFFM, BarbosaFilho JM 2003. Natural products inhibitors of ovarian neoplasia. Phytomedicine 10: 221-232.

Simopoulos AP, Norman HA, Gillaspy JE, Duke JA 1992. Common purslane: a source of omega-3 fatty acids and antioxidants. J Amer Coll Nutr 11: 374-382.

Simpson DJ, Baqar MR, Lee TH 1977. Fine structure and carotenoid composition of the fibrillar chromoplasts of Asparagus officinalis. Ann Bot (London) 41: 1101-1108.

Skaltsa M, Couladi M, Philianos S, Singh M 1987. Phytochemical study of the leaves of Ocimum 
sanctum. Fitoterapia 58: 286.

Souleles C, Vayas K 1986. Constituents of the leaves of Aesculus hippocastanum. Fitoterapia 57: 201-202.

Steinmetz KA, Potter JD 1996. Vegetables, fruit, and cancer prevention: a review. J Amer Diet Ass 96: 1027-1039.

Strocchi A, Lercker G, Bonaga G, Maye A 1977. Composition of papaya seed oil. Riv Ital Sostanze Grasse 5: 429.

Subbarayan C, Cama HR 1964. Carotenoid in Carica papaya (papaya fruit). Indian J Chem 2: 451.

Sun JM 1994. Process for extracting natural beta-carotene from carrot. Patent-Faming Zhuanli Shenqing Gongkai Shuomingshu-1,092,063:9pp.

Takagi S 1985. Determination of green leaf carotenoids by HPLC. Agr Biol Chem 49: 1211-1213.

Takaichi S, Tsuji K, Hanada S, Matsuura K, Shimada K 1995. A novel carotenoid glucoside ester in green filamentous bacteria. Photosynth: Light Biosphere Proc Int Photosynth Congr 10th 4: 127-130.

Takaichi S, Wang ZY, Umetsu M, Nozawa T, Shimada K, Madigan MT 1997. New carotenoids from the thermophilic green sulfur Bacterium chlorobium tepidum: 1',2'-dihydro-gamma-carotene, 1',2'dihydrochlorobactene, and-chlorobactene glucoside ester, and the carotenoid composition of different strains. Arch Microbiol 168: 270-276.

Takenaka H, Takahashi H, Hayashi K, Ben-Amotz A 1993. Protective effect of Dunaliella bardawil on waterimmersion-induced stress in rats. Planta Med 59: 421-424.

Tamas V, Popescu V 1985. Carotenoid pigments in some varieties and populations of Lolium perenne. Stud Ceret Biochim 28: 152-154.

Tanaka Y, Yamada S, Sameshima M 1992. Novel apocarotenoid apoastacenal isolated from nudibranch eggmasses. Nippon Suisan Gakkaishi 58: 1549.

Tangen K, Bjoernland T 1981. Observations on pigments and morphology of Gyrodinium aureolum Hulburt, a marine dinoflagellate containing 19'-hexanoyloxyfucoxanthin as the main carotenoid. J Plankton Res 3: 389-401.

Tirimanna ASL 1981. Study of the carotennoid pigments of Bixa orellana L. seeds by thin layer chromatography. Mikrochim Acta 2: 11-16.

Topuriya Li 1990. Pigments of the plastids and flavonoids of the leaves of Carica papaya. Chem Nat Comp 26: 98-99.

Toth G, Szabolics J 1981. Occurrence of some monocis-isomers of asymmetric C-40-carotenoids. Phytochemistry 20: 2411-2415.

Toth K, Madl F, Koch L, Matits S, Koch B, Schlich P 1995. Beta-carotene isolation from plants. Patent-Hung Teljes-69-643:15pp.

Trofimova EP 1977. Some wild food plants of Tadzhikistan as sources of vitamins. Izv Akad Nauk Tadzh Ssr Otd Biol Nauk 1: 43-48.

Tzakou O, Loukis A, Philianos S 1988. Constituents of petrol extract of Galium helderichii. Fitoterapia 59: 254.

Uddin A, Chowdhury AR 1982. Chemical examination of leaves of Solanum khasianum. Int J Crude Drug Res 20: 139-140.

Ugaz OL, Fort AD 1984. Triterpenes from Vernonia baccharoides. Bol Soc Quim Peru 50: 258-261.

Valadon LRG, Mummery RS 1972. Carotenoids of rowan berries. Ann Bot (London) 361: 471-474.

Valadon LRG, Mummery RS 1974. Carotenogenesis in Verticillium agaricinum in response to nicotine and to CPTA. Microbios 10: 97.

Valadon LRG, Mummery RS 1975. Carotenoids of floral parts and of the spadix of Arum maculatum. $Z$ Pflanzenphysiol 75: 88.

Van Breemen RB 1996. Innovations in carotenoid analysis using LC/MS. Anal Chem 68: 299-304.

Vanhaelen M 1973. Identification of carotenoids in Arnica montana. Planta Med 23: 308-311.

Vega PJ, Balaban MO, Sims CA, O’keefe SF, Cornell JA 1996. Supercritical carbon dioxide extraction efficiency for carotenes from carrots by RSM. J Food Sci 61: 757-759.

Vitsaropoulou EV, Philianos S 1981. The constituents of the leaves of Sarcopoterium spinosum (L.) Spach. (Rosaceae). Plant Med Phytother 15: 16-20.

Wang JM, Liao DS, Li QG, Wang ZL 1992. Chemical constituents and function of Pyracantha roemer pigments. Shipin Kexue (Beijing) 15: 4-6.

Williams BL, Goodwin TW 1965. The terpenoids of tissue cultures of paul's scarlet rose. Phytochemistry 4: 81-88.

Winde E, Echaust I, Hansel R 1961. Verbena officinalis. Occurrence of adenosine and beta-carotene. Question of the "verbenin" of Kuwajima. Arch Pharm (Weinheim) 29: 220-229.

Wu JT, Huang TC 1975. Biosystematic studies of Formosan salvia. Taiwania 20: 77.

$\mathrm{Wu}$ TS 1991. Murrayamine-A,-B,-C and (+)-mahanine, carbozole alkaloids from Murraya euchrestifolia. Phytochemistry 30: 1048-1051.

Wu Y, Perry AK, Klein BP 1992. Vitamin C and beta-carotene in fresh and frozen green beans and broccoli in a simulated system. J Food Qual 15: 87-96.

Yadav S, Ralhan PK, Singh SP 1987. Qualitative distribution pattern of carotenoids in three selected Gymnosperms. Curr Sci 56: 354-359.

Yajima T, Hirano M, Okada J 1993. Phaffia rhodozyma mutants for commercial manufacture of carotenoids. PatentJapan Kokai Tokkyo Koho-05 168,465:6pp.

Yamamoto HY 1964. Comparison of the carotenoids in yellowand red-fleshed Carica papaya. Nature 201: 1049.

Yen GC, Wu SC, Du PD 1996. Extraction and identification of antioxidant components from the leaves of mulberry (Morus alba L.). J Agr Food Chem 44: 1687-1690.

Zechmeister L, Cholnoky L 1932a. The petal pigment of Calendula officinalis. Matematik Termeszettud Ertesito 4: 181.

Zechmeister L, Cholnoky LV 1932b. The pigment of marigold (Calendula officinalis) floral lycopin. Hoppe-Seyler's Z Physiol Chem 20: 26.

Zherebin YUI, Kolesnik AA 1984. Carotenoids of the grape. Chem Nat Comp 20: 110-111.

Zhmyrko TG, Gigienova EI, Umarov AU 1978. Vitamins of the oil of the fruit of Hippophae rhamnoides. Chem Nat Comp 14: 259-263.

Zhou W, Xu Y, Zhao X, Wang J 1984. Preparation and application of provitamin concentrate from pine needles. Linchan Hua Hsueh Yu Gong Yi 44: 28-32.

Zorikov PS, Burii TP 1974. Content of carotene and vitamins $\mathrm{E}$ and $\mathrm{C}$ in some far-eastern plants. Deposited Doc. 73274: 18. 